\title{
Proper actions of lattices on contractible manifolds
}

\author{
Mladen Bestvina and Mark Feighn *
}

October 30,2018

\begin{abstract}
Every lattice $\Gamma$ in a connected semi-simple Lie group $G$ acts properly discontinuously by isometries on the contractible manifold $G / K$ ( $K$ a maximal compact subgroup of $G$ ). We prove that if $\Gamma$ acts on a contractible manifold $W$ and if either

1) the action is properly discontinuous, or

2) $W$ is equipped with a complete Riemannian metric, the action is by isometries and with unbounded orbits, $G$ is simple with finite center and rank $>1$,

then $\operatorname{dim} W \geq \operatorname{dim} G / K$.
\end{abstract}

\section{Introduction}

Let $G$ be a connected semi-simple Lie group and $K$ a maximal compact subgroup of $G$. It is a theorem of Malcev Mal45] and Iwasawa [wa49 that the homogeneous space $G / K$ (even without the assumption of semi-simplicity) is a contractible manifold (see [Hoc65, Theorem XV.3.1] and also Mos55 for the case when $G$ has finitely many components). When $G$ has finite center, $G / K$ is a symmetric space of noncompact type. Every lattice $\Gamma \subset G$ acts properly discontinuously on $G / K$. The main theorem of this paper is:

Theorem 1.1. If $\Gamma$ acts properly discontinuously (by homeomorphisms) on a contractible manifold $W$, then $\operatorname{dim} W \geq \operatorname{dim} G / K$.

By contrast, $\Gamma$ often acts properly discontinuously on a contractible complex of smaller dimension than that of $G / K$. The minimal dimension of such a complex, for torsion-free $\Gamma$, is the cohomological dimension $\operatorname{cd} \Gamma$ of $\Gamma$ (with the possible exception of the case $\mathrm{cd} \Gamma=2$, but such examples are unlikely to occur

${ }^{*}$ The authors gratefully acknowledge the support by the National Science Foundation. 
among lattices in connected Lie groups) and it has been computed in the case of arithmetic lattices by Borel and Serre BS73 - the difference $\operatorname{dim} G / K-\operatorname{cd} \Gamma$ is equal to the $\mathbb{Q}$-rank of $\Gamma$. In many specific cases such complexes were found by Ash [Ash77.

Theorem 1.1 answers a question of Shmuel Weinberger and Kevin Whyte. The bulk of the proof is dedicated to the special case when $G$ is a real linear algebraic group and $\Gamma$ is an arithmetic lattice in $G$. The reduction to this case is presented in Section 3. Next, we present the proof for the case when $\Gamma=S L_{n}(\mathbb{Z}) \subset G=S L_{n}(\mathbb{R})$, focusing on $n=3$. This case contains most of the ideas needed in general and has the advantage that the proof does not use the jargon of algebraic groups. The general proof in the arithmetic case is in Section 10 and there are more examples preceding and following this section. The discussion of what happens when the assumption of proper discontinuity is replaced by the assumption that the action is isometric is in Section 4 .

The method of proof is based on [BKK]. For $m \geq 0$ an $m$-obstructor complex is one that does not embed into $\mathbb{R}^{m}$ for homological reasons. For the precise definition, see [BKK]. We will only need the fact that the join

$$
S^{a} * S_{+}^{k_{1}} * S_{+}^{k_{2}} * \cdots * S_{+}^{k_{r}}
$$

is an $m$-obstructor complex for

$$
m=a+k_{1}+k_{2}+\cdots+k_{r}+2 r-1
$$

and that

$$
S_{+}^{k_{1}} * S_{+}^{k_{2}} * \cdots * S_{+}^{k_{r}}
$$

is an $n$-obstructor complex for

$$
n=k_{1}+k_{2}+\cdots+k_{r}+2 r-2
$$

where $S_{+}^{k}$ denotes the $k$-sphere with an extra point added, and each sphere $S^{k}$ is triangulated as the join of 0 -spheres. The above fact then follows from the Join Lemma of [BKK].

When $L$ is a finite simplicial complex define the (open) cone on $L$ as

$$
\operatorname{cone}(L)=L \times[0, \infty) / L \times\{0\} .
$$

If $X$ is a proper metric space, a map $H: \operatorname{cone}(L) \rightarrow X$ is proper expanding if it is proper and whenever $\sigma$ and $\tau$ are disjoint simplices of $L$ then

$$
d(H(\sigma \times[t, \infty)), H(\tau \times[t, \infty))) \rightarrow \infty
$$


as $t \rightarrow \infty$. We also say that the cones on $\sigma$ and $\tau$ diverge (under $H$ ) if (1) holds.

When $\Gamma$ is any discrete group of type $F_{\infty}$ (i.e. there is an Eilenberg-MacLane complex $K(\Gamma, 1)$ with finitely many cells in each dimension), we write

$$
\text { " } L \subset \partial \Gamma "
$$

if there is an isometric, properly discontinuous, cocompact action of $\Gamma$ on a proper metric space $X$ and a proper expanding map cone $(L) \rightarrow X$. When $\Gamma$ is a lattice in $G$, for $X$ we will frequently use a bounded neighborhood of a $\Gamma$-orbit in $G$ or $G / K$ (equipped with $G$-invariant Riemannian metrics). The formal definition of the concept " $L \subset \partial \Gamma$ " in BKK is more complicated and applies to any finitely generated group, but all groups we consider in this paper will be of type $F_{\infty}$ and for such groups the definition given above is equivalent to the general definition (see [BKK, Remark 11]).

The obstructor dimension obdim $\Gamma$ of $\Gamma$ is defined in BKK to be $m+2$ where $m$ is the largest integer such that " $L \subset \partial \Gamma$ " for some $m$-obstructor complex $L$. Passing to subgroups of finite index and quotients by finite normal subgroups does not change obdim.

The main theorem of [BKK] is:

Theorem 1.2. BKK If obdim $\Gamma=m+2$ then $\Gamma$ cannot act properly discontinuously on a contractible manifold of dimension $<m+2$.

The theorem we actually prove in this paper is:

Theorem 1.3. obdim $\Gamma=\operatorname{dim} G / K$.

Example 1.4. If $\Gamma$ is a uniform lattice in $G$ and if $G$ has finite center, then $\Gamma$ acts cocompactly on the symmetric space $G / K$ which is a contractible manifold of dimension say $m+2$ with a complete Riemannian metric of nonpositive curvature (see e.g. Hel78, Theorem V.3.1]). Thus the exponential map gives a proper expanding map $\mathbb{R}^{m+2}=\operatorname{cone}\left(S^{m+1}\right) \rightarrow G / K$ (where $S^{m+1}$ is viewed as the unit tangent space at a point of $G / K)$. Thus " $S^{m+1} \subset \partial \Gamma$ " and obdim $\Gamma=$ $\operatorname{dim} G / K$, proving the theorem in this case. The same argument works for groups of the form $G \times A$ where $G$ is as above and $A$ is connected abelian.

Theorem 1.1 follows from Theorem 1.3 and Theorem 1.2. It is clear from Theorem 1.2 that obdim $\Gamma \leq \operatorname{dim} G / K$. To prove the reverse inequality we have to construct an $m$-obstructor complex $L$ with " $L \subset \partial \Gamma$ " and $m=\operatorname{dim} G / K-2$.

We would like to thank Dragan Miličić for his help with the theory of Lie groups and algebraic groups and to Misha Kapovich and Bruce Kleiner for several useful conversations. 


\section{A fibration lemma}

A discrete version of the following lemma is in BKK. The proof, although it closely parallels the discrete version, is more transparent.

Lemma 2.1. Let $G$ be a connected Lie group and $H \subset G$ a closed subgroup such that the homogeneous space $Q=G / H$ is contractible. The metric on $G$ is chosen to be invariant under left translations, on $Q$ so that the quotient map $\pi: G \rightarrow Q$ is a Lipschitz map, and on $H$ it is the subspace metric. Suppose that $H$ and $Q$ admit proper expanding maps of cones on finite complexes $K_{H}, K_{Q}$ respectively. Then $G$ admits a proper expanding map of the cone on the join $K_{H} * K_{Q}$. Moreover, it can be arranged that the image of this map is contained in the $\pi$-preimage of the image of cone $\left(K_{Q}\right)$.

Proof. The quotient map $\pi: G \rightarrow Q$ is a fibration, and since $Q$ is contractible there is a continuous section $s: Q \rightarrow G$. Let $\alpha: \operatorname{cone}\left(K_{H}\right) \rightarrow H$ and $\beta$ : cone $\left(K_{Q}\right) \rightarrow Q$ be the given maps. Define

$$
f: \operatorname{cone}\left(K_{H} * K_{Q}\right)=\operatorname{cone}\left(K_{H}\right) \times \operatorname{cone}\left(K_{Q}\right) \rightarrow G
$$

by

$$
f(x, y)=\alpha(x) \cdot s \beta(y) .
$$

We have the commutative diagram

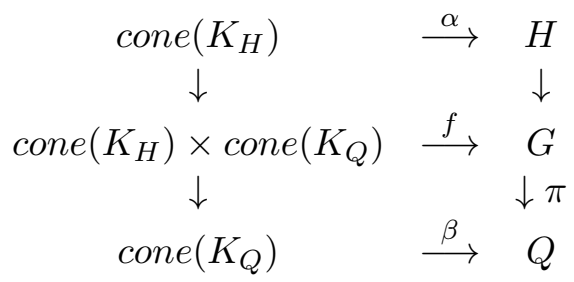

Claim. $f$ is a proper map.

Indeed, let $\left(x_{i}, y_{i}\right)$ be a sequence in $\operatorname{cone}\left(K_{H}\right) \times \operatorname{cone}\left(K_{Q}\right)$ leaving every compact set. If the sequence $\pi f\left(x_{i}, y_{i}\right)=\beta\left(y_{i}\right) \in Q$ leaves every compact set, the same is true for $f\left(x_{i}, y_{i}\right) \in G$. Otherwise, after passing to a subsequence, we may assume that the sequence $\pi f\left(x_{i}, y_{i}\right)=\beta\left(y_{i}\right) \in Q$ stays in a compact set $D \subset Q$. Then $s \beta\left(y_{i}\right)$ stays in the compact set $s(D)$. Since $\beta$ is a proper map, the sequence $y_{i} \in \operatorname{cone}\left(K_{Q}\right)$ stays in a compact set, and thus the sequence $x_{i} \in \operatorname{cone}\left(K_{H}\right)$ leaves every compact set. Since $\alpha$ is a proper map, we see that the sequence $f\left(x_{i}, y_{i}\right)=\alpha\left(x_{i}\right) \cdot s \beta\left(y_{i}\right)$ leaves every compact set.

Claim. If $\sigma=\sigma_{H} * \sigma_{Q}$ and $\tau=\tau_{H} * \tau_{Q}$ are disjoint simplices of $K_{H} * K_{Q}$, then $f \mid \operatorname{cone}(\sigma)$ and $f \mid$ cone $(\tau)$ diverge. 
Indeed, let $\left(x_{i}, y_{i}\right)$ and $\left(x_{i}^{\prime}, y_{i}^{\prime}\right)$ be sequences in $\operatorname{cone}\left(\sigma_{H}\right) \times \operatorname{cone}\left(\sigma_{Q}\right)$ and $\operatorname{cone}\left(\tau_{H}\right) \times \operatorname{cone}\left(\tau_{Q}\right)$ respectively, leaving every compact set. Since $\pi$ is a Lipschitz map, if one of two sequences $\pi f\left(x_{i}, y_{i}\right)=\beta\left(y_{i}\right)$ and $\pi f\left(x_{i}^{\prime}, y_{i}^{\prime}\right)=\beta\left(y_{i}^{\prime}\right)$ leaves every compact set in $Q$, then $d_{Q}\left(\beta\left(y_{i}\right), \beta\left(y_{i}^{\prime}\right)\right) \rightarrow \infty\left(\operatorname{since} \beta \mid \operatorname{cone}\left(\sigma_{Q}\right)\right.$ and $\beta \mid$ cone $\left(\tau_{Q}\right)$ diverge) and consequently $d_{G}\left(f\left(x_{i}, y_{i}\right), f\left(x_{i}^{\prime}, y_{i}^{\prime}\right)\right) \rightarrow \infty$. Now assume that both sequences $\beta\left(y_{i}\right)$ and $\beta\left(y_{i}^{\prime}\right)$ are contained in a fixed compact set $D \subset Q$. Then we have

$$
\begin{array}{r}
d_{G}\left(f\left(x_{i}, y_{i}\right), f\left(x_{i}^{\prime}, y_{i}^{\prime}\right)\right)=d_{G}\left(\alpha\left(x_{i}\right) \cdot s \beta\left(y_{i}\right), \alpha\left(x_{i}^{\prime}\right) \cdot s \beta\left(y_{i}^{\prime}\right)\right)= \\
d_{G}\left(1, s \beta\left(y_{i}\right)^{-1} \alpha\left(x_{i}\right)^{-1} \alpha\left(x_{i}^{\prime}\right) s \beta\left(y_{i}^{\prime}\right)\right)
\end{array}
$$

Since $s \beta\left(y_{i}\right)$ and $s \beta\left(y_{i}^{\prime}\right)$ stay in a compact set and

$$
d_{H}\left(1, \alpha\left(x_{i}\right)^{-1} \alpha\left(x_{i}^{\prime}\right)\right)=d_{H}\left(\alpha\left(x_{i}\right), \alpha\left(x_{i}^{\prime}\right)\right) \rightarrow \infty
$$

it follows that

$$
d_{G}\left(1, \alpha\left(x_{i}\right)^{-1} \alpha\left(x_{i}^{\prime}\right)\right) \rightarrow \infty
$$

and

$$
d_{G}\left(1, s \beta\left(y_{i}\right)^{-1} \alpha\left(x_{i}\right)^{-1} \alpha\left(x_{i}^{\prime}\right) s \beta\left(y_{i}^{\prime}\right)\right) \rightarrow \infty .
$$

Remark 1. Another reasonable choice of a metric on $H$ would be an $H$-invariant Riemannian metric. Say two proper metrics $d_{1}$ and $d_{2}$ on a space $X$ are equivalent if there is a homeomorphism $\varphi:[0, \infty) \rightarrow[0, \infty)$ such that $d_{2}(x, y) \leq$ $\varphi\left(d_{1}(x, y)\right)$ and $d_{1}(x, y) \leq \varphi\left(d_{2}(x, y)\right)$ for all $x, y \in X$. Any proper expanding map cone $(L) \rightarrow X$ with respect to $d_{1}$ is also proper expanding with respect to $d_{2}$. For example, any two Riemannian $G$-invariant metrics on $G$ or on $G / K$ are equivalent. Various choices of metrics on $H$ as indicated above are all equivalent.

The following consequence can be viewed as the analog of Theorem 1.1 in the context of nilpotent groups. Of course, the first three statements are well known (see Rag72]).

Corollary 2.2. Let $\Gamma$ be a lattice in a simply connected nilpotent Lie group $N$. Then

- $\Gamma$ is cocompact in $N$,

- $N$ is diffeomorphic to Euclidean space of dimension $m+2$, say,

- $N$ contains no nontrivial compact subgroups, and 
- " $S^{m+1} \subset \partial \Gamma "$ and hence obdim $\Gamma=\operatorname{dim} N$.

Proof. Let $Z$ be the center of $N$. By Rag72, Proposition 2.17] the intersection $Z \cap \Gamma$ is a lattice in $Z$. All claims now follow by induction on $\operatorname{dim} N$ from the exact sequence

$$
1 \rightarrow Z \rightarrow N \rightarrow N / Z \rightarrow 1
$$

and Lemma 2.1.

Example 2.3. Let $N_{n}$ be the group of real upper-triangular matrices with 1's on the diagonal, let $N_{n}(\mathbb{Z})$ be the lattice in $N_{n}$ consisting of matrices with integral entries, and let $m+2=1+2+\cdots+(n-1)$ denote the number of matrix positions above the diagonal. Then

$$
\text { "S } S^{m+1}=*_{i=1}^{m+2} S^{0} \subset \partial N_{n}(\mathbb{Z}) " .
$$

Specifically, if we regard the cone on $S^{m+1}$ as $\mathbb{R}^{m+2}$ with a coordinate for every position above the diagonal, and we regard the cone on a simplex of $S^{m+1}$ as the set of matrices in $H_{n}$ where the entries in the specified positions have specified signs and the other entries above the diagonal are 0, then this map cone $\left(S^{m+1}\right) \rightarrow H_{n}$ is proper and expanding.

Another application of Lemma 2.1 is to semi-simple groups with infinite center, e.g. $\widetilde{S L_{2}(\mathbb{R})}$.

Corollary 2.4. Suppose $G$ is a connected semi-simple Lie group with infinite center $Z$, and let $\Gamma \subset G$ be a lattice such that $\Gamma \cap Z$ has finite index in $Z$ so that $\Gamma /(\Gamma \cap Z)$ is a lattice in $G / Z$. Then $\operatorname{obdim} \Gamma \geq \operatorname{obdim}(\Gamma / \Gamma \cap Z)+\operatorname{rank}(Z)$.

Proof. Let $K$ be a maximal compact subgroup in $G / Z$. Now apply Lemma 2.1 to the preimage $H \subset G$ of $K$ in $G$.

\section{Reduction to arithmetic lattices}

We will review the terminology and basic facts about algebraic and arithmetic groups in Section 10. The bulk of the paper is dedicated to the proof of the following theorem:

Theorem 3.1. Let $G \subset G L_{n}(\mathbb{C})$ be a semi-simple linear algebraic group defined over $\mathbb{Q}$ and let $\Gamma_{\mathbb{Z}}=G \cap G L_{n}(\mathbb{Z}) \subset G_{\mathbb{R}}$ be the standard arithmetic lattice in the group of real points. Then obdim $\Gamma_{\mathbb{Z}}=\operatorname{dim} G_{\mathbb{R}} / K$.

In this section we deduce Theorem 1.3 from Theorem 3.1. We first prove another special case. 
Theorem 3.2. Let $G$ be a semi-simple real algebraic group and $\Gamma$ a lattice in $G$. Then obdim $\Gamma_{\mathbb{Z}}=\operatorname{dim} G_{\mathbb{R}} / K$.

Proof. We can assume that the component of the identity of $G_{\mathbb{R}}$ has no compact factors. Further, using the Product Lemma [BKK], we may assume that $\Gamma$ is an irreducible lattice in $G_{\mathbb{R}}$. If the real rank of $G$ is $>1$ then the celebrated theorem of Margulis [Zim84, Theorem 6.1.2] says that $\Gamma$ is an arithmetic lattice (with respect to some $\mathbb{Q}$-structure on $G$ ) and the theorem follows from Theorem 3.1. Now suppose that the real rank of $G$ is 1 . Then the symmetric space $G / K$ is real, complex, or quaternionic hyperbolic space or the Cayley plane (see [Hel78, Chapter X]). If $\Gamma$ is a uniform lattice acting cocompactly on $G / K$, then the statement follows from Example 1.4, so we may assume that $\Gamma$ is a nonuniform lattice. If $G / K$ is the real hyperbolic space $\mathbb{H}^{m+2}$ let $P$ be a maximal parabolic subgroup of $\Gamma$. Then $P$ is commensurable to $\mathbb{Z}^{m+1}$ and there is a proper expanding map of the cone on $S^{m}$ into a horosphere stabilized by $P$. Adding a ray that diverges from this horosphere but stays within a bounded distance from a $\Gamma$-orbit produces a proper expanding map from the cone on $S_{+}^{m}$ and shows that obdim $(\Gamma)=m+2$. If $G / K$ is the complex hyperbolic space of complex dimension $d=\frac{m+2}{2}$, then a maximal parabolic subgroup $P$ of $\Gamma$ is a lattice in the Heisenberg group $N$ that is a central extension $1 \rightarrow \mathbb{R} \rightarrow N \rightarrow$ $\mathbb{C}^{d-1} \rightarrow 1$. By Corollary 2.2 there is a proper expanding map of the cone on $S^{m}$ into a horosphere stabilized by $P$. The argument now follows as in the real case. If $G / K$ is quaternionic hyperbolic space or the Cayley plane, then $\Gamma$ is an arithmetic lattice [GS92] and the claim again follows from Theorem 3.1.

Proof of Theorem 1.3. First suppose that the center $Z$ of $G$ is finite. If necessary, replace $G$ by $G / Z$ so that the center is trivial. Then $G$ has the structure of (the identity component of) a linear real algebraic group (see e.g. [Zim84, Proposition 3.1.6]) and the proof is reduced to Theorem 3.2.

Now suppose that the center $Z$ of $G$ is infinite. Again we may assume that $G$ has no compact factors. Then by [Rag72, 5.17] $Z \Gamma$ is a discrete subgroup of $G$, so [Rag72, 1.13] (with $H=Z$ ) implies that $\Gamma \cap Z$ has finite index in $Z$. It now follows from Corollary 2.4 and the centerless case applied to the lattice $\Gamma /(Z \cap \Gamma) \subset G / Z$ that

$$
\operatorname{obdim} \Gamma \geq \operatorname{rank} Z+\operatorname{obdim} \Gamma /(Z \cap \Gamma)=\operatorname{rank} Z+\operatorname{dim}(G / Z) / C
$$

where $C$ is a maximal compact subgroup of $G / Z$. It remains to show that

$$
\operatorname{dim} G / K=\operatorname{rank} Z+\operatorname{dim}(G / Z) / C .
$$

After passing to finite covers, we may assume that $C=T \times C^{\prime}$ where $T$ is a torus and $C^{\prime}$ is a simply connected compact group (see e.g. Kna96, Theorem 
4.29]). The preimage of $C$ in $G$ decomposes as $\mathbb{R}^{m} \times T^{\prime} \times C^{\prime}$ where $T^{\prime}$ is a torus and $m=\operatorname{rank} Z$. Then $K$ can be identified with $T^{\prime} \times C^{\prime}$, so we have

$$
\begin{array}{r}
\operatorname{dim} G / K=\operatorname{dim} G-\operatorname{dim} K=\operatorname{dim}(G / Z)-\left(\operatorname{dim} C^{\prime}+\operatorname{dim} T^{\prime}\right)= \\
\operatorname{dim}(G / Z)-\operatorname{dim} C+m=\operatorname{dim}(G / Z)-\operatorname{dim} C+\operatorname{rank} Z
\end{array}
$$

\section{Isometric Actions}

We need the following fact from the theory of Lie groups.

Lemma 4.1. If $H$ is a connected noncompact Lie group, then there is a representation $\rho: H \rightarrow G L_{N}(\mathbb{R})$ such that the closure of the image $\overline{\rho(H)}$ is noncompact.

Proof. Assume that the adjoint representation has precompact image. Then the Lie algebra $\mathfrak{h}$ of $H$ admits an ad-invariant inner product and hence breaks up as the direct sum of simple and abelian Lie algebras. The simple summands have compact type, so the integral subgroup $C \subset H$ corresponding to the sum of all simple summands is compact, as well as normal in $H$. The quotient $H / C$ is a noncompact connected abelian group and it therefore maps onto $\mathbb{R}$.

Note that the group $G L_{N}(\mathbb{R})$ above can be replaced by $P G L_{N+1}(\mathbb{C})$ since there is a proper map $G L_{N}(\mathbb{R}) \rightarrow P G L_{N+1}(\mathbb{C})$.

Theorem 4.2. Suppose that $M$ is a contractible manifold equipped with a complete Riemannian metric. Let $G$ be a connected simple Lie group of rank $>1$ with finite center and let $\Gamma$ be a lattice in $G$. If $\operatorname{dim} M<\operatorname{dim} G / K$ then every isometric action of $\Gamma$ on $M$ has a bounded orbit. In particular, if the metric on $M$ is $C A T(0)$, then every isometric action of $\Gamma$ on $M$ has a global fixed point.

Proof. The isometry group $\operatorname{Isom}(M)$ of $M$ is a Lie group. Let $\varphi: \Gamma \rightarrow$ $\operatorname{Isom}(M)$ be the given action, and denote by $H$ the closure of $\varphi(\Gamma)$. Then $H$ is also a Lie group. If $H$ has infinitely many components, then the MargulisKazhdan theorem [Zim84, Theorem 8.1.2] implies that $\varphi: \Gamma \rightarrow \operatorname{Isom}(M)$ has finite kernel and the image of $\varphi$ is closed. It follows that the action of $\Gamma$ on $M$ is properly discontinuous, contradicting the assumption $\operatorname{dim} M<\operatorname{dim} G / K$ and Theorem 1.1. Now suppose that $H$ has only finitely many components. After passing to a subgroup of $\Gamma$ of finite index, we may assume that $H$ is connected. If $H$ is compact, then the $H$-orbits, and hence $\Gamma$-orbits, are bounded. Thus assume that $H$ is noncompact. Then $H$ admits a representation $\rho: H \rightarrow P G L_{N+1}(\mathbb{C})$ 
whose image has noncompact closure (see Lemma 4.1). We can arrange in addition that $\rho$ is trivial on the finite central subgroup $\varphi(\Gamma \cap Z(G)$ ) (by applying Lemma 4.1 to $H / \varphi(\Gamma \cap Z(G)))$. By the Margulis' super-rigidity Zim84, Theorem 5.1.2] (applied to an algebraic $\mathbb{R}$-group that has $G / Z(G)$ as the identity component [Zim84, Proposition 3.1.6] and to the lattice $\Gamma /(\Gamma \cap Z(G)))$ there is a continuous extension $\tilde{\rho}: G \rightarrow P G L_{N+1}(\mathbb{C})$. The kernel $\operatorname{Ker}(\tilde{\rho}) \neq G$ is a normal subgroup of $G$ and is therefore contained in the center. It follows from Got48 (see also [Hel78, Exercise II.D.4]) that $\tilde{\rho}(G)$ is a closed subgroup of $P G L_{N+1}(\mathbb{C})$, thus the image of $\varphi$ is closed and discrete and $H$ cannot be connected, contradiction. The last sentence follows from the Cartan fixed point theorem BH99, Corollary II.2.8].

If $G$ has rank 1, one can say the following. When $G / K$ is real or complex hyperbolic space there exist lattices with positive first Betti number, and they of course act isometrically on $\mathbb{R}$ with unbounded orbits. In the real case this is a result of Millson Mil76 and the complex case a result of Kazhdan (see [BW80, Corollary VIII 5.9]). The cases of quaternionic hyperbolic space or the Cayley plane are open. By Property T, lattices in these cases have trivial first Betti number. One possible scenario would be to have infinite quotients of smaller cohomological dimension that act discretely on a contractible manifold of smaller dimension.

Question: Do lattices in $S p(n, 1)$ have infinite quotients of strictly smaller (virtual or rational) cohomological dimension?

If $G$ is not simple, but only semi-simple (and with finite center), then $G / K$ decomposes (see Hel78, Proposition V.4.2, Proposition VIII.5.5]) as the direct product $X_{1} \times X_{2} \times \cdots \times X_{m}$ of irreducible symmetric spaces and an irreducible lattice $\Gamma \subset G$ acts isometrically on each $X_{i}$ with unbounded orbits. After replacing (without loss of generality!) $G$ by a finite cover, $G$ also decomposes as $G_{1} \times G_{2} \times \cdots \times G_{m} \times C$ where $C$ is a compact group, $G_{i}$ are simple and $G_{i} / K_{i}=X_{i}$.

Theorem 4.3. Suppose that $G=G_{1} \times G_{2} \times \cdots \times G_{m}(m>1)$ is the product of noncompact simple Lie groups with finite center and that an irreducible lattice $\Gamma \subset G$ acts isometrically on a piecewise Riemannian contractible manifold $M$ with $\operatorname{dim} M<\operatorname{dim} X_{i}$ for all $i=1,2, \cdots, m$. Then all $\Gamma$-orbits are bounded.

Proof. Follow the proof above verbatim until the extension $\tilde{\rho}: G \rightarrow P G L_{N+1}(\mathbb{C})$ is considered. Now the kernel is a closed normal subgroup and its Lie subalgebra can be assumed, after reordering the factors, to be equal to the Lie subalgebra of $G_{1} \times \cdots \times G_{i}$ for some $1 \leq i<m$ (if the kernel is discrete the argument 
concludes the same way). It follows that the product

$$
G \rightarrow G_{1} \times \cdots \times G_{i} \times P G L_{N+1}(\mathbb{C})
$$

of projections and of $\tilde{\rho}$ has finite kernel. Now Got48] again implies that the image of this map is closed, and hence the diagonal action of $\Gamma$ on $X_{1} \times X_{2} \times$ $\cdots \times X_{i} \times M$ is properly discontinuous. Theorem 1.1 now implies that $\operatorname{dim} M \geq$ $\operatorname{dim} X_{i+1}+\cdots+\operatorname{dim} X_{m} \geq \operatorname{dim} X_{m}$.

Remark 2. The metric on $M$ can be allowed to be more general than Riemannian in both theorems above. The proof only requires that the metric is proper and that $\operatorname{Isom}(M)$ is a Lie group. The latter is satisfied e.g. if the Hausdorff dimension of the metric is $<\operatorname{dim} M+2$ by RS97. Hilbert-Smith conjecture implies that $\operatorname{Isom}(M)$ is a Lie group for any metric on $M$.

\section{$5 \quad$ Examples}

In the examples that follow (before and after the proof of Theorem 3.1) $G$ will be a semi-simple group of matrices, $\Gamma$ a lattice in $G$ and $K$ a maximal compact subgroup of $G$. If the dimension of the symmetric space $G / K$ is $m+2$, the goal is to construct a proper expanding map cone $(L) \rightarrow G$ of an $m$-obstructor complex $L$ in $G$ whose image is in a bounded neighborhood of $\Gamma$. To compute the dimension of $X=G / K$ we will make use of the Iwasawa decomposition $G=K A N$ that implies $\operatorname{dim} X=\operatorname{dim} A+\operatorname{dim} N$.

\section{$6 \quad S L_{3}(\mathbb{Z})$}

We now discuss the case $\Gamma=S L_{3}(\mathbb{Z})$ in detail. Here $G=S L_{3}(\mathbb{R}), K=S O_{3}$, and the symmetric space $X=G / K$ is 5 -dimensional. We will construct the 3 -obstructor complex $L=S_{+}^{0} * S_{+}^{1}$ in $\partial \Gamma$.

\subsection{The cuspidal complex $C$}

We will focus on the subgroup $B \subset \Gamma$ consisting of upper-triangular matrices with 1's on the diagonal and on the 6 conjugates of $B$ obtained by simultaneously permuting rows and columns. As we know (see Example 2.3) each copy of $B$ has a natural 2-sphere at infinity. To understand how different 2 -spheres fit together we define the simplicial complex $C$. Its vertices are the 6 off-diagonal positions in a $3 \times 3$ matrix. A vertex $i j$ can be viewed as an oriented arrow from a point labeled $i$ to a point labeled $j(i, j \in\{1,2,3\})$. A collection of 
vertices of $C$ spans a simplex if there is a conjugate of $B$ as above that has non-zero entries in all the corresponding positions. In the language of arrows, the condition is that there are no oriented cycles. See Fig. 1.

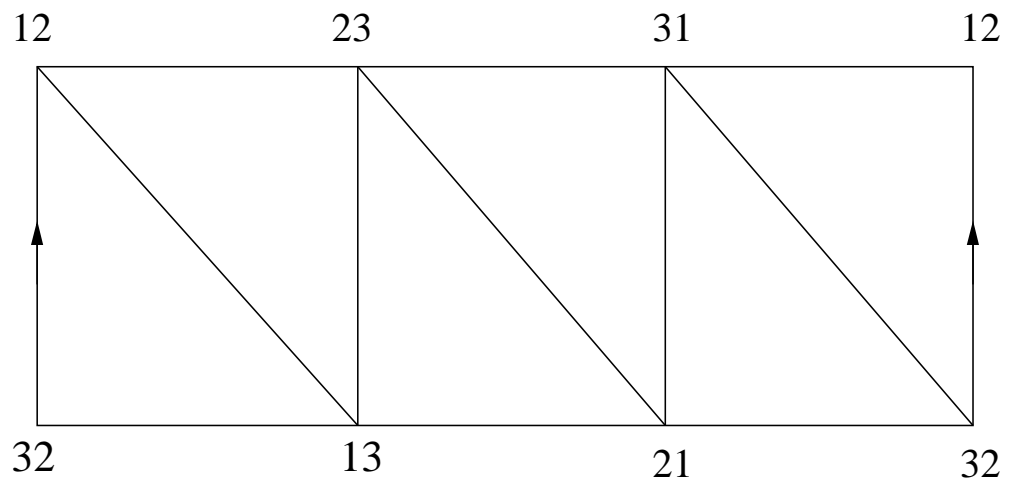

Figure 1: The complex $C$ is an annulus with a drum triangulation

\subsection{The complex $S C$}

We now define a functorial procedure that assigns a complex $S C$ to the complex $C$. Each simplex will be replaced by the sphere of the same dimension. A vertex $v$ of $C$ corresponds to two vertices, thought of as $v+$ and $v-$, in $S C$. Thus the vertex set $S C^{(0)}$ of $S C$ is the vertex set $C^{(0)}$ of $C$ crossed with $\{+,-\}$. There is a natural projection $\pi: S C^{(0)} \rightarrow C^{(0)}$ that forgets the sign. A collection of vertices of $S C$ spans a simplex iff $\pi$ is injective on this collection and the image in $C^{(0)}$ is the vertex set of a simplex. The projection $\pi$ extends to a simplicial map $\pi: S C \rightarrow C$. A simplex of dimension $k$ in $C$ has precisely $2^{k+1}$ lifts to $S C$ and the full preimage is a $k$-sphere triangulated as the $(k+1)$-fold join of 0 -spheres.

\subsection{Two lemmas}

Lemma 6.1 (Lemma A). SC contains a 3-obstructor complex L.

Lemma 6.2 (Lemma B). " $L \subset \partial S L_{3}(\mathbb{Z})$ "

Remark 3. We don't know if "SC $\subset \partial S L_{n}(\mathbb{Z})$ ". 
We will prove Lemma B for $S L_{n}(\mathbb{Z})$ in the next section. For Lemma A, consider the subcomplex $L$ of $S C$ formed by the full preimage (which is a 2sphere) of one of the triangles in $C$, say $<12,23,13>$; add to it an equatorial disk which is half of the sphere $\pi^{-1}(<13,23,21>)$; finally add the vertex $32+$ and edges joining it to the north and south poles and the center of the equatorial disk. See Fig. 2.

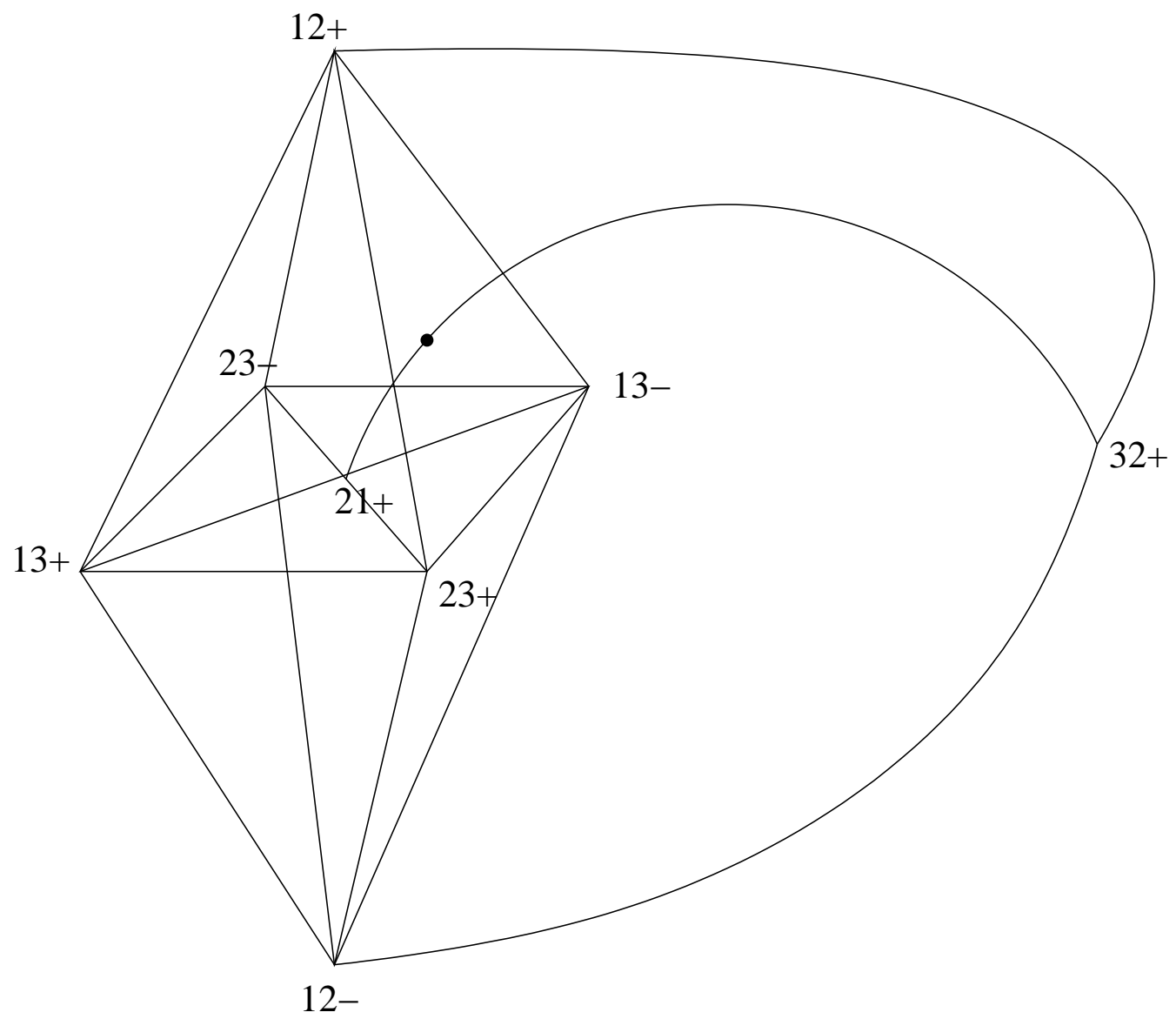

Figure 2: A 3-obstructor subcomplex $L \subset S C$ nearly embedded in $\mathbb{R}^{3}$. The Van Kampen intersection point is indicated.

This subcomplex $L$ is the join of the disjoint union of the circle $\pi^{-1}(<$ $13,23>$ ) and the vertex $32+$ and the three points $12+, 12-, 21+$. By the Join Lemma of BKK, $L$ is a 3-obstructor complex. This shows obdim $S L_{3}(\mathbb{Z})=5$. 


\section{$7 \quad \Gamma=S L_{n}(\mathbb{Z})$}

Here $G=S L_{n}(\mathbb{R}), A$ is the group of diagonal matrices in $G$, and $N$ is the group of upper triangular matrices in $G$ with 1's on the diagonal. Thus $\operatorname{dim} X=$ $\operatorname{dim} A N=(1+2+\cdots+n)-1=\frac{n^{2}}{2}+\frac{n}{2}-1$. We let

$$
L=S_{+}^{0} * S_{+}^{1} * \cdots * S_{+}^{n-2}
$$

which is an $m$-obstructor complex for $m=0+1+\cdots+(n-2)+2(n-2)=$ $\frac{n^{2}}{2}+\frac{n}{2}-3$.

We again define the complex $C$ with $n^{2}-n$ vertices, one for every offdiagonal position of an $n \times n$ matrix, and a collection of vertices spans a simplex iff the corresponding positions are all above the diagonal after a simultaneous permutation of rows and columns. Let $S C$ be the corresponding complex whose vertices are signed vertices of $C$ and whose simplices are lifts of simplices of $C$.

Lemma 7.1 (Lemma A). SC contains the m-obstructor complex L.

Lemma 7.2 (Lemma B). " $L \subset \partial S L_{n}(\mathbb{Z})$ "

Proof of Lemma A. The sphere $S^{k-2}$ is the full preimage of the $(k-2)$-simplex $<1 k, 2 k, \cdots,(k-1) k>$ of $C$ and we add the vertex $k(k-1)+$ to form $S_{+}^{k}$. It is straightforward to check that the subcomplex of $S C$ spanned by the described vertices is precisely a copy of $L$.

\subsection{Lemma B - discussion}

We first define a map from the cone on $S C$ into $G=S L_{n}(\mathbb{R})$. A simplex of $S C$ corresponds to a collection of positions with signs and the first guess might be that the cone on such a simplex is sent to the subset of $G$ having 1's on the diagonal, entries of appropriate sign in the positions corresponding to the vertices of the simplex, and 0's in all other positions. The problem with this rule is that cones on disjoint simplices don't diverge. For example (letting $R$ be large), let

$$
A=\left[\begin{array}{lll}
1 & R & 1 \\
0 & 1 & 1 \\
0 & 0 & 1
\end{array}\right]
$$

and

$$
B=\left[\begin{array}{ccc}
1 & 0 & -R \\
0 & 1 & 0 \\
0 & 1 & 1
\end{array}\right]
$$


Then

$$
A^{-1} B=\left[\begin{array}{ccc}
1 & -1 & -1 \\
0 & 0 & -1 \\
0 & 1 & 1
\end{array}\right]
$$

so $A$ and $B$ are bounded distance apart. Instead, we separate the positions below diagonal from the positions above the diagonal, thus obtaining two matrices, one upper-triangular and the other lower-triangular. We then send the point in the cone to the product of the two matrices. For example, the cone on $<23+, 31+>$ is thought of not as the set of matrices

$$
\left[\begin{array}{lll}
1 & 0 & 0 \\
0 & 1 & x \\
y & 0 & 1
\end{array}\right]
$$

with $x, y \geq 0$ but as the set of matrices

$$
\left[\begin{array}{lll}
1 & 0 & 0 \\
0 & 1 & x \\
0 & 0 & 1
\end{array}\right]\left[\begin{array}{lll}
1 & 0 & 0 \\
0 & 1 & 0 \\
y & 0 & 1
\end{array}\right]=\left[\begin{array}{rrr}
1 & 0 & 0 \\
x y & 1 & x \\
y & 0 & 1
\end{array}\right]
$$

with $x, y \geq 0$.

In the example above, $A$ is already upper-triangular and would represent the image of the chosen point in the cone on $<12+, 23+, 13+>$, but the matrix $B$ would be replaced by the product

$$
\left[\begin{array}{ccc}
1 & 0 & -R \\
0 & 1 & 0 \\
0 & 0 & 1
\end{array}\right]\left[\begin{array}{lll}
1 & 0 & 0 \\
0 & 1 & 0 \\
0 & 1 & 1
\end{array}\right]=\left[\begin{array}{ccc}
1 & -R & -R \\
0 & 1 & 0 \\
0 & 1 & 1
\end{array}\right]
$$

If this matrix is denoted by $B^{\prime}$, then we compute that

$$
A^{-1} B^{\prime}=\left[\begin{array}{ccc}
1 & -R-1 & -1 \\
0 & 0 & -1 \\
0 & 1 & 1
\end{array}\right]
$$

so $A$ and $B^{\prime}$ are far apart. In this case it can be shown that this first guess for the map suffices to prove that " $L \subset \partial S L_{n}(\mathbb{Z})$ "; however, in general we find it easier to deal with the map corresponding to separating the upper-triangular from the lower-triangular part.

Lemma 7.3. For every $n$ there is a function $\phi:[0, \infty) \rightarrow[0, \infty)$ with the following properties. Let $\Lambda=\left(l_{i j}\right), \Lambda^{\prime}=\left(l_{i j}^{\prime}\right)$ be two lower triangular matrices with 1's on the diagonal, and let $U, U^{\prime}$ be two upper-triangular matrices with 1 's on the diagonal. Assume: 
- If $l_{i j} \neq 0$ or if $l_{i j}^{\prime} \neq 0$ then either $i=j$ or $i=j+1$.

- $l_{j+1, j} l_{j+1, j}^{\prime}=0$ for all $j$.

If at least one of $\Lambda, \Lambda^{\prime}$ contains an entry of absolute value $>\phi(R)$ then $S=$ $(U \Lambda)^{-1} U^{\prime} \Lambda^{\prime}$ has an entry of absolute value $>R$.

Proof. We can write $U \Lambda=U^{\prime} \Lambda^{\prime} S$. Assume that the statement is false, and let $U_{k}, U_{k}^{\prime}, \Lambda_{k}, \Lambda_{k}^{\prime}, S_{k}$ be a sequence of examples with all entries of $S$ bounded by $R$ and some entries of $\Lambda$ or $\Lambda^{\prime}$ going to infinity. By passing to a subsequence we may assume that all entries converge in $[-\infty, \infty]$. Call a position of a matrix 'small' if the corresponding entries stay bounded and otherwise call it 'large'. For example, all positions of $S$ are small. Note that by Cramer's Rule (det $S=1$ ) all positions of $S^{-1}$ are also small. For simplicity, we drop the subscripts from $\Lambda_{k}, U_{k}, \cdots$.

Consider the lowest nonzero off-diagonal entry $x$ of $\Lambda$ or $\Lambda^{\prime}$, i.e. $l_{j+1, j}$ or $l_{j+1, j}^{\prime}$ such that $l_{m+1, m}=l_{m+1, m}^{\prime}=0$ for all $m>j$. There are two cases:

Case 1: The position of $x$ is small. We can replace $x$ by 0 without affecting other entries of the same matrix by multiplying on the right by the elementary lower-triangular matrix that has entry $-x$ in the position $j+1, j$. We can compensate this operation by either multiplying $S$ on the right by the same matrix (in case that the entry $x$ belongs to $\Lambda$ ) or by multiplying $S$ on the left by the inverse of this matrix (in case $x$ belongs to $\Lambda^{\prime}$ ). All entries of the new matrix $S$ are still small (though not bounded by $R$ perhaps). Now proceed to the next lowermost off-diagonal entry of $\Lambda$ or $\Lambda^{\prime}$.

Case 2: The position of $x$ is large. Say $x$ belongs to $\Lambda$ (the other case is analogous). We write

$$
U^{\prime-1} U \Lambda=\Lambda^{\prime} S
$$

The right hand side is obtained from the matrix $S$ with small entries by applying elementary row operations in which a multiple of the $p^{\text {th }}$ row is added to the row below for certain $p<j$. Therefore all rows bellow row $j$ of the matrix $\Lambda^{\prime} S$ are equal to the corresponding entries of the small matrix $S$.

The left hand side is obtained from $\Lambda$ by applying elementary row operations in which a multiple of a row is added to some row above. It follows that the entry of $U^{\prime-1} U \Lambda$ in position $(j+1, j)$ is $x$, which is large. Contradiction

Proof of Lemma $B$. Let $U \Lambda$ and $U^{\prime} \Lambda^{\prime}$ be two matrices representing points in cones on disjoint simplices of $L$. Assuming that the two points are far away from the cone point, we have to show that the distance between $U \Lambda$ and $U^{\prime} \Lambda^{\prime}$ is large. If one of $\Lambda, \Lambda^{\prime}$ has a large entry, this follows from Lemma 7.3. If both $\Lambda$ and $\Lambda^{\prime}$ have small entries, then $U \Lambda$ is close to $U$, while $U^{\prime} \Lambda^{\prime}$ is close to $U^{\prime}$, 
so we must show that $U$ and $U^{\prime}$ are far apart. In this case $U$ and $U^{\prime}$ will have large entries, so the statement follows from Example 2.3.

We can see more concretely how the cone on $L$ is mapped to $G$. The sphere $S^{p-2}$ is identified with the unit sphere in $\mathbb{R}^{p-1}$, which in turn is mapped to $G$ via

$$
\left(x_{1}, x_{2}, \cdots, x_{p-1}\right) \mapsto\left[\begin{array}{ccccc}
1 & & x_{1} & \\
& 1 & & x_{2} & \\
& & & & \\
& & & & \\
& & & & \\
& & & & 1
\end{array}\right]
$$

and the extra point determines the ray of positive numbers in the $(p, p-1)$ position.

$$
8 \Gamma=S L_{n}(\mathbb{Z}[\sqrt{2}])
$$

$\mathcal{O}=\mathbb{Z}[\sqrt{2}])$ is a lattice in $\mathbb{R}^{2}$ under the embedding $a+b \sqrt{2} \mapsto(a+b \sqrt{2}, a-b \sqrt{2})$ and likewise $\Gamma$ is a lattice in $G=S L_{n}(\mathbb{R}) \times S L_{n}(\mathbb{R})$ (with the same map applied to each entry). Note that $\mathcal{O}$ is a ring and the group of units $\mathcal{O}^{*}$ has rank 1 (e.g. $1+\sqrt{2}$ has infinite order). The symmetric space is $X \times X$ with $X=S L_{n}(\mathbb{R}) / S O_{n}$. We define $L$ as the join

$$
L=S^{n-2} * S_{+}^{1} * S_{+}^{3} * \cdots * S_{+}^{2 n-3} .
$$

Then $L$ is an $m$-obstructor complex for $m=(n-1)+1+3+\cdots+(2 n-3)+$ $2(n-2)=n^{2}+n-4$ while $X \times X$ has dimension $n^{2}+n-2$.

We define the map $\Psi$ : cone $(L) \rightarrow G$ analogously to the $S L_{n}(\mathbb{Z})$ construction, with a new feature that there are diagonal entries different from 1 this time.

Fix some $p \in\{2,3, \cdots, n\}$ and consider the subgroup $\Gamma_{p}$ of $\Gamma$ consisting of matrices that have any entry from $\mathbb{Z}[\sqrt{2}]$ in positions $(1, p),(2, p), \cdots,(p-1, p)$, 1 's on the diagonal, and 0 's in the remaining positions.

$$
\left[\begin{array}{ccccc}
1 & & & x_{1} & \\
& 1 & & x_{2} & \\
& & & \\
& & x_{p-1} & \\
& & 1 & \\
& & & \\
& & & & 1
\end{array}\right]
$$


To this subgroup (isomorphic to $\mathbb{Z}^{2 p-2}$ ) we associate the subgroup $G_{p}$ of $G$ isomorphic to $\mathbb{R}^{2 p-2}$ consisting of the set of pairs $(A, B)$ of matrices as above with real entries. Note that $\Gamma_{p}$ is a cocompact lattice in $G_{p}$. We view $G_{p}$ as the cone on $S^{2 p-3}$ with the cone on a simplex of $S^{2 p-3}$ corresponding to the subset of $G_{p}$ where certain entries of $A$ and $B$ above the diagonal and in column $p$ are nonnegative, certain others are nonpositive, and the remaining entries are 0 .

Next, we define a ray $R_{p}$ in $G$ to consist of pairs of matrices $(\Lambda, \Lambda)$ with $\Lambda$ having 0's above the diagonal, 1's on the diagonal, entry $x \geq 0$ in position $(p, p-1)$ and 0's in all other positions. This ray is also within a bounded distance from $\Gamma$. We identify the cone on $S_{+}^{2 p-2}$ with $G_{p_{+}}:=G_{p} \cup R_{p}$.

Finally, we define $\Gamma_{0}$ to consist of diagonal matrices in $\Gamma$, and we define $G_{0}$ to consist of pairs $\left(D, D^{-1}\right) \in G$ where $D$ is a diagonal matrix with positive diagonal entries. We have that $\Gamma_{0}$ is an abelian group of rank $n-1, G_{0} \cong \mathbb{R}^{n-1}$, and $G_{0}$ is within a bounded neighborhood of $\Gamma_{0}$. We identify $G_{0}$ with the cone on $S^{n-2}$ where the cone on a simplex of $S^{n-2}$ corresponds to the pairs $\left(D, D^{-1}\right)$ with certain diagonal entries of $D$ bounded below by 1 , certain others bounded above by 1 , and the remaining diagonal entries equal to 1 .

We now define the map $\Psi$ : cone $(L) \rightarrow G$. We are identifying cone $(L)$ with $G_{0} \times G_{2+} \times \cdots \times G_{n+}$. Let $\left(\left(D, D^{-1}\right),\left(A_{2}, B_{2}\right), \cdots,\left(A_{n}, B_{n}\right)\right) \in$ cone $(L)$. From this data we first form 3 pairs of matrices:

- diagonal pair $\left(D, D^{-1}\right)$,

- lower-triangular pair $(\Lambda, \Lambda)$ - it is formed by superimposing (equivalently, adding entries below the diagonal) all lower-triangular pairs $\left(\Lambda_{p}, \Lambda_{p}\right)$ appearing in the sequence $\left(A_{p}, B_{p}\right)$, and

- upper-triangular pair $(U, V)$ - it is formed by superimposing (equivalently, adding entries above the diagonal) all upper-triangular pairs $\left(A_{p}, B_{p}\right)$ appearing in the above sequence.

We define the image under $\Psi$ of the given sequence to be the pair

$$
\left(U D \Lambda, V D^{-1} \Lambda\right) \in G \text {. }
$$

Lemma 8.1 (Lemma B). " $L \subset \partial S L_{n}(\mathbb{Z}[\sqrt{2}])$ ".

The proof is similar to the proof in the case of $S L_{n}(\mathbb{Z})$ except for the added complication of diagonal matrices. It will not be given here and we appeal to the general case of Lemma B given is Section 11 . 


\section{$9 \quad \Gamma=S L_{n}(\mathcal{O})$}

Here $\mathcal{O}$ is the ring of integers in a number field $k$. Let $r$ be the number of real places of $k$ and $s$ the number of complex-conjugate places. Then, as an abelian group, $\mathcal{O} \simeq \mathbb{Z}^{r+2 s}$. The group $\mathcal{O}^{*}$ of units is a finitely generated group of rank $r+s-1$. For example, when $\mathcal{O}=\mathbb{Z}[i]$ then $r=0$ and $s=1$, and when $\mathcal{O}=\mathbb{Z}[\sqrt{2}]$ then $r=1$ and $s=0$. $\mathcal{O}$ has $r$ embeddings in $\mathbb{R}$ and $s$ embeddings in $\mathbb{C}$, and there is an induced diagonal embedding $\Gamma \subset G=$ $S L_{n}(\mathbb{R})^{r} \times S L_{n}(\mathbb{C})^{s}$ and $\Gamma$ is a lattice there. The symmetric space associated to $S L_{n}(\mathbb{C})$ has dimension equal to the dimension of $A(=n-1)$ plus the dimension of $N(=2(1+2+\cdots+(n-1)))$, so it equals $n^{2}-1$. Thus the dimension of the symmetric space of $G$ is

$$
r\left(\frac{n^{2}}{2}+\frac{n}{2}-1\right)+s\left(n^{2}-1\right)
$$

We set

$$
L=\underset{\substack{S_{+}^{(r+2 s)+(r+s-1)-1} * \\ S_{+}^{(n-1)(r+2 s)+(r+s-1)-1}}}{S_{+}^{2(r+2 s)+(r+s-1)-1} * S_{+}^{3(r+2 s)+(r+s-1)-1} * \cdots *}
$$

which is an $m$-obstructor complex for

$$
\begin{aligned}
m & =(1+2+\cdots+(n-1))(r+2 s)+(n-1)(r+s-2)+2(n-2) \\
& =r\left(\frac{n^{2}}{2}+\frac{n}{2}-1\right)+s\left(n^{2}-1\right)-2
\end{aligned}
$$

The complex $S_{+}^{(p-1)(r+2 s)+(r+s-1)-1}$ is identified with the unit sphere in the space $\mathbb{R}^{(p-1)(r+2 s)+(r+s-1)}$ union a ray. The Euclidean space is realized (as an abelian group with that rank) by upper triangular matrices with entries $(1, p),(2, p), \cdots,(p-1, p)$ from $\mathcal{O}$, entry $(p, p)$ from $\mathcal{O}^{*}$, entry $(1,1)$ the inverse of entry $(p, p)$, other diagonal entries are 1 , and all other entries are 0 . The ray is realized by matrices with diagonal entries 1 , and positive real entry in position $(p, p-1)$.

\section{Proof of Theorem 3.1}

In this section we consider the case of arithmetic lattices. We use Rag72], [Bor91, and Bor69] as general references on algebraic and arithmetic groups. Let $G \subset G L_{N}(\mathbb{C})$ denote a connected semi-simple linear algebraic group defined over $\mathbb{Q}$. If $k$ is a subring of $\mathbb{C}$ (usually $\mathbb{Z}, \mathbb{Q}, \mathbb{R}$, or $\mathbb{C}$ ) denote by $G_{k}$ the group $G \cap G L_{N}(k)$ of $k$-points. It is a theorem of Borel-Harish-Chandra BHC62 that 
$G_{\mathbb{Z}}$ is a lattice in $G_{\mathbb{R}}$. This is the standard arithmetic lattice. Let $S$ be the maximal torus in $G$ which is split over $\mathbb{Q}$ and let $M$ be the largest connected subgroup of the centralizer $Z(S)$ which is anisotropic, i.e. does not have any nontrivial $\mathbb{Q}$-split tori (in other words, the $\mathbb{Q}$-rank of $M$ is 0 ). We also have that the component of the identity $Z(S)^{0}=S \cdot M$, i.e. the multiplication map $S \times M \rightarrow Z(S)^{0}$ is surjective and has finite kernel. Further, $M$ is reductive [Bor91, IV.13.17 Corollary 2], i.e. after a finite cover it decomposes as the product of a torus and a semi-simple $\mathbb{Q}$-group.

There is the usual decomposition of the Lie algebra $\mathfrak{g}$ of $G$ :

$$
\mathfrak{g}=\mathfrak{g}_{0} \oplus \bigoplus_{\alpha \in \Phi} \mathfrak{g}_{\alpha}
$$

into root spaces. Each $\alpha \in \Phi$ is a rational character $\alpha: S \rightarrow G L_{1}(\mathbb{C})$ and $\mathfrak{g}_{\alpha}$ is the associated root space. It is customary to use additive notation in the group of characters. Choose an ordering on $\Phi$ and denote by $\Phi^{+}$the set of positive roots and by $\Delta$ the set of simple roots (those roots not expressible as sums of other roots in $\Phi^{+}$). The cardinality $r$ of $\Delta$ is equal to the dimension of $S$ and is called the $\mathbb{Q}$-rank of $G$. Lattice $G_{\mathbb{Z}}$ is cocompact in $G_{\mathbb{R}}$ if and only if the $\mathbb{Q}$-rank is 0 (this statement holds even for reductive groups). Unlike in the case of algebraically closed fields, the root spaces $\mathfrak{g}_{\alpha}$ may have dimension $>1$ and the root system $\Phi$ may not be reduced (we may have $0 \neq \alpha, 2 \alpha \in \Phi$ ). Of course, $\Phi$ might not be irreducible (the Dynkin diagram could be disconnected). Every irreducible component of $\Phi$ is either reduced (i.e. it is of type $A_{n}(n \geq 1), B_{n}$ $(n \geq 3), C_{n}(n \geq 2), D_{n}(n \geq 4), E_{6}, E_{7}, E_{8}, F_{4}$, or $\left.G_{2}\right)$ or unreduced (i.e. it is of type $\left.B C_{n}(n \geq 1)\right)$. See e.g. Kna96.

The subalgebra of $\mathfrak{g}$ corresponding to $Z(S)^{0}$ is precisely $\mathfrak{g}_{0}$.

Lemma 10.1. $M_{\mathbb{Z}}$ acts cocompactly and properly discontinuously on a contractible manifold $X_{M}$ and there is a sphere " $S_{M} \subset \partial M_{\mathbb{Z}}$ " with $\operatorname{dim} S_{M}=$ $\operatorname{dim} X_{M}-1$.

Proof. $M_{\mathbb{Z}}$ is a lattice in $M_{\mathbb{R}}$ by BHC62, Theorem 9.4]. It is a cocompact lattice since the $\mathbb{Q}$-rank of $M$ is 0 . The manifold $X_{M}$ can be taken to be the product of the symmetric space of the semi-simple factor of $M$ and of the Euclidean factor corresponding to $T_{\mathbb{R}} / K_{T}$ (real points in the torus modulo maximal compact subgroup). See Example 1.4.

The Lie algebra of a minimal $\mathbb{Q}$-parabolic subgroup $P$ is $\mathfrak{g}_{0} \oplus \bigoplus_{\alpha \in \Phi^{+}} \mathfrak{g}_{\alpha}$ and $P=Z(S)^{0} \cdot U$ where $U$ is the connected nilpotent subgroup with Lie algebra $\bigoplus_{\alpha \in \Phi^{+}} \mathfrak{g}_{\alpha}$. 
Lemma 10.2. $P_{\mathbb{Z}}=P \cap G L_{N}(\mathbb{Z})$ acts cocompactly and properly discontinuously on a contractible manifold $X_{P}$ and there is a sphere " $S_{P} \subset \partial P_{\mathbb{Z}}$ " with $\operatorname{dim} S_{P}=\operatorname{dim} X_{P}-1$ and $\operatorname{dim} X_{P}=\operatorname{dim} U_{\mathbb{R}}+\operatorname{dim} X_{M}$. Moreover, $\operatorname{dim} X_{P}=\operatorname{dim} G_{\mathbb{R}} / K-r$.

Proof. Note that $S$ intersects $G_{\mathbb{Z}}$ in a finite subgroup. After passing to a finite cover, we have a split exact sequence

$$
1 \rightarrow U \rightarrow P / S \rightarrow Z(S)^{0} / S \rightarrow 1
$$

and $Z(S)^{0} / S$ is a quotient of $M$ with finite kernel. The image of $P_{\mathbb{Z}}$ is a lattice in $P_{\mathbb{R}} / S_{\mathbb{R}}$. A maximal compact subgroup $K^{\prime}$ of $Z(S)_{\mathbb{R}}^{0} / S_{\mathbb{R}}$ lifts to a maximal compact subgroup of $P_{\mathbb{R}} / S_{\mathbb{R}}$. We set $X_{P}=\left(P_{\mathbb{R}} / S_{\mathbb{R}}\right) / K^{\prime}$ and apply Lemma 2.1 to $K^{\prime} \rtimes U_{\mathbb{R}} \subset P_{\mathbb{R}} / S_{\mathbb{R}}$. To prove the last statement, recall [BS73] that $G_{\mathbb{Z}}$ acts cocompactly and properly discontinuously on a contractible manifold with corners whose interior can be identified with $G_{\mathbb{R}} / K$ and $P_{\mathbb{Z}}$ is the stabilizer of a lowest dimensional stratum, which is a contractible manifold and has codimension $r$ (and is really a copy of $X_{P}$ ).

We now note that if the $\mathbb{Q}$-rank $r=0$ the theorem follows from Example 1.4. From now on we will assume that $r \geq 1$.

We now state some lemmas. For every $\alpha \in \Delta$ set $\hat{\alpha}=2 \alpha$ if $2 \alpha \in \Phi$ and otherwise set $\hat{\alpha}=\alpha$. Let $\hat{\Delta}=\{\hat{\alpha} \mid \alpha \in \Delta\}$.

Lemma 10.3. Let $\Phi$ be a (possibly unreduced, possibly not irreducible) root system, and let $\Delta$ be the set of simple roots (with respect to some ordering). There is an ordering of the set $\hat{\Delta}$ with the following properties. Let $\hat{\alpha} \in \hat{\Delta}$. Suppose that the elements of $\hat{\Delta}$ that precede $\hat{\alpha}$ in the order are labeled by one of the letters " $U$ " or " $D$ ", and also label $\hat{\alpha}$ itself by " $D$ ". We refer to the elements of $\hat{\Delta}$ labeled " $D$ " as D-nodes, and those labeled " $U$ " as U-nodes. Then there exist $\sigma, \mu \in \Phi \cup\{0\}$ such that:

1. $\mu-\sigma=\hat{\alpha}$,

2. the difference $\sigma-\phi$ between $\sigma$ and any $\phi \in \Phi \cup\{0\}$ is not a positive multiple of a D-node,

3. the difference $\phi-\sigma$ between any $\phi \in \Phi \cup\{0\}$ is not a positive multiple of a U-node.

Label "U" means that it is not possible to go "up" from $\sigma$ along the simple root and reach a root or 0 and, similarly, "D" stands for "down". We postpone the proof of this lemma until the end of Section 11. 
Lemma 10.4. 1. If $v \in \mathfrak{g}_{\alpha}, w \in \mathfrak{g}_{\beta}$ then $[v, w] \in \mathfrak{g}_{\alpha+\beta}$ (the latter is defined to be 0 unless $\alpha+\beta \in \Phi \cup\{0\})$.

2. Suppose that $\alpha, \beta, \alpha+\beta \in \Phi \cup\{0\}$ and $\alpha \neq 0$. Then there exist $v \in \mathfrak{g}_{\alpha}$ and $w \in \mathfrak{g}_{\beta}$ such that $[v, w] \neq 0$.

Proof. Both statements are well-known over $\mathbb{C}$ (see e.g. Kna96, Proposition 2.5] for (1) and [FH91, Claim 21.19] for (2)). (1) is proved the same way over $\mathbb{Q}$, and statement $(2)$ over $\mathbb{Q}$ follows by decomposing each root space into 1dimensional subspaces which are root spaces with respect to a maximal torus that contains $S$ (but is split only over $\mathbb{C}$, not over $\mathbb{Q}$ ).

\section{Lemma 10.5 .}

$\exp (X) Y \exp (-X)=Y+[X, Y]+\frac{1}{2 !}[X,[X, Y]]+\frac{1}{3 !}[X,[X,[X, Y]]]+\cdots$

Lemma 10.6. Rag79, Corollary 10.14] Let $M$ be an algebraic group defined over $\mathbb{Q}$ and let $\rho: M \rightarrow G L(V)$ be a homomorphism defined over $\mathbb{Q}$ into the general linear group of a vector space defined over $\mathbb{Q}$. If $\Gamma \subset M$ is an arithmetic lattice in $M$ and if $L$ is a lattice in $V_{\mathbb{Q}}$ then there is a finite index subgroup $\Gamma^{\prime}$ of $\Gamma$ such that $\rho\left(\Gamma^{\prime}\right)$ preserves $L$.

For each $\hat{\alpha} \in \hat{\Delta}$ choose a rational vector $\zeta_{\hat{\alpha}} \in \mathfrak{g}_{-\hat{\alpha}}$ such that

$$
\left[\zeta_{\hat{\alpha}}, \cdot\right]: \mathfrak{g}_{\mu} \rightarrow \mathfrak{g}_{\sigma}
$$

is nonzero whenever $\sigma, \mu \in \Phi \cup\{0\}$ are such that $\mu-\sigma=\hat{\alpha}$. That such a vector exists follows from Lemma 10.4 and it can be taken to be a rational vector by perturbing (the set of bad choices is contained in a finite union of proper subspaces).

Let $\alpha_{1}, \cdots, \alpha_{r}$ be the simple roots in $\Delta$ ordered so that $\hat{\alpha}_{1}, \hat{\alpha}_{2}, \cdots, \hat{\alpha}_{r}$ is the ordering of $\hat{\Delta}$ from Lemma 10.3. Every root in $\Phi^{+}$is an integral combination with nonnegative coefficients of the simple roots. For $i=1, \cdots, r$ denote by $\Phi_{i}^{+}$ the set of positive roots obtained as nonnegative integral linear combinations of simple roots $\alpha_{1}, \alpha_{2}, \cdots, \alpha_{i}$ and involving $\alpha_{i}$ with a positive coefficient. Note that if $\alpha, \alpha^{\prime} \in \Phi_{i}^{+}$and if $\alpha+\alpha^{\prime}$ is a root, then $\alpha+\alpha^{\prime} \in \Phi_{i}^{+}$. It follows that $\mathfrak{n}_{i}=\bigoplus_{\alpha \in \Phi_{i}^{+}} \mathfrak{g}_{\alpha}$ is a nilpotent subalgebra of $\mathfrak{g}$. The integral subgroup $N_{i}=\exp \left(\mathfrak{n}_{i}\right)$ is nilpotent and the intersection $N_{i} \cap G_{\mathbb{Z}}$ is a lattice in $N_{i} \cap G_{\mathbb{R}}$. Thus by Corollary 2.2 we have an expanding map $C_{i} \rightarrow N_{i} \cap G_{\mathbb{R}}$ from the cone $C_{i}$ on a sphere $S_{i}$ with

$$
\operatorname{dim} C_{i}=\operatorname{dim} N_{i} \cap G_{\mathbb{R}}=\sum_{\alpha \in \Phi_{i}^{+}} \operatorname{dim} \mathfrak{g}_{\alpha}
$$


where $\operatorname{dim} \mathfrak{g}_{\alpha}$ is the complex dimension (or equivalently the real dimension of the real points of $\mathfrak{g}_{\alpha}$ ). The $N_{i}$ 's play the role of the "column groups" in our examples. The reader will note that the maps $C_{i} \rightarrow N_{i} \cap G_{\mathbb{R}}$ have been constructed as homeomorphisms and it will do no harm (and it will simplify notation) to omit the name of the map and simply identify $C_{i}$ with $N_{i}$.

Similarly, for $i=1,2, \cdots, r$, consider the abelian subalgebra $\mathfrak{g}_{-\hat{\alpha}_{i}}$. By $P_{i}=\exp \left(\mathfrak{g}_{-\hat{\alpha}_{i}}\right)$ denote the associated integral subgroup and again by Corollary 2.2 we know that $P_{i} \cap G_{\mathbb{Z}}$ is a cocompact lattice in $P_{i} \cap G_{\mathbb{R}}$. The latter is a noncompact group and we define a proper embedding $R_{i}:[0, \infty) \rightarrow P_{i} \cap G_{\mathbb{R}}$ (whose image we also denote by $R_{i}$ ) by

$$
R_{i}(t)=\exp \left(t \zeta_{\hat{\alpha}_{i}}\right)
$$

The $P_{i}$ 's play the role of the subdiagonal positions in our examples.

We now define

$$
C=C_{M} \times\left(C_{1} \cup R_{1}\right) \times\left(C_{2} \cup R_{2}\right) \times \cdots \times\left(C_{r} \cup R_{r}\right) .
$$

This is the open cone on a finite complex $L$ obtained by taking joins of a sphere (for $C_{M}$ ) and spheres with a point added. We take the join triangulation on $L$. Define also a map

$$
\Psi: C \rightarrow G_{\mathbb{R}}
$$

as follows. Let $\left(d, z_{1}, z_{2}, \cdots, z_{r}\right) \in C$. Then each $z_{i}$ is either equal to some $x_{i} \in$ $C_{i}$ or to some $y_{i} \in R_{i}$. Say $z_{i}=x_{i} \in C_{i}$ for $i=i_{1}, i_{2}, \cdots, i_{k}$ and $z_{i}=y_{i} \in R_{i}$ for $i=j_{1}, j_{2}, \cdots, j_{l}$ where we assume $i_{1}<i_{2}<\cdots<i_{k}, j_{1}<j_{2}<\cdots<j_{l}$ and $k+l=r$. Then set

$$
\Psi\left(d, z_{1}, z_{2}, \cdots, z_{r}\right)=x_{i_{1}} x_{i_{2}} \cdots x_{i_{k}} d y_{j_{1}} y_{j_{2}} \cdots y_{j_{l}} .
$$

Note that the image of $\Psi$ is contained in a bounded neighborhood of $G_{\mathbb{Z}}$ in $G_{\mathbb{R}}$ - this is true for the components $R_{i}, C_{i}$ and $C_{M}$ by construction and remains true after taking pointwise products of such neighborhoods.

Remark 4. Suppose $A$ and $B$ are $3 \times 3$ elementary matrices with nonzero entries in positions $(1,2)$ and $(2,3)$ respectively. Then $A B$ is a matrix with a nonzero $(1,3)$ position, while $B A$ is obtained from $A$ and $B$ by "superposition" (as in our examples). This explains why we have to carefully arrange different components of the map $\Psi$.

The proof of Theorem 3.1 is now reduced to the following two lemmas.

Lemma A. $L$ is an m-obstructor complex with $m=\operatorname{dim} X_{G}-2$.

Lemma B. $\Psi$ is proper and expanding 
Proof of Lemma A. We have

$$
L=L_{M} * L_{1} * L_{2} * \cdots * L_{r}
$$

where $L_{M}$ is the sphere of dimension $\operatorname{dim} X_{M}-1$ and $L_{i}$ is the disjoint union of a sphere of dimension $\sum_{\alpha \in \Phi_{i}^{+}} \operatorname{dim} \mathfrak{g}_{\alpha}-1$ and a point. Thus $L_{M}$ is a $\left(\operatorname{dim} X_{M}-2\right)$ obstructor complex and $L_{i}$ is a $\sum_{\alpha \in \Phi_{i}^{+}} \operatorname{dim} \mathfrak{g}_{\alpha}-1$-obstructor complex. The Join Lemma implies that $L$ is an $m$-obstructor complex for

$$
\begin{array}{r}
m=\left(\operatorname{dim} X_{M}-2\right)+\sum_{i=1}^{r}\left(\sum_{\alpha \in \Phi_{i}^{+}} \operatorname{dim} \mathfrak{g}_{\alpha}-1\right)+2 r= \\
\operatorname{dim} X_{M}+\sum_{\alpha \in \Phi^{+}} \operatorname{dim} \mathfrak{g}_{\alpha}+r-2=\operatorname{dim} X_{P}+r-2=\operatorname{dim} G_{\mathbb{R}} / K-2
\end{array}
$$

where the last two equalities follow from Lemma 10.2 and the observation that $\operatorname{dim} U_{\mathbb{R}}=\sum_{i=1}^{r} \sum_{\alpha \in \Phi_{i}^{+}} \operatorname{dim} \mathfrak{g}_{\alpha}$.

\section{Proof of Lemma B}

Choose two disjoint simplices of $L$ and a sequence of points in their cones. To simplify notation, we omit the subscripts corresponding to the sequence counters. We then have $\left(d, z_{1}, \cdots, z_{r}\right)$ and $\left(d^{\prime}, z_{1}^{\prime}, \cdots, z_{r}^{\prime}\right)$. Say the indices of $z_{i}$ corresponding to nontrivial points in the rays are $j_{1}<j_{2}<\cdots<j_{l}$ and of $z_{i}^{\prime}$ they are $k_{1}<k_{2}<\cdots<k_{q}$. The two sets are disjoint. For concreteness, assume $j_{l}>k_{q}$. If possible, pass to a subsequence such that the point $z_{j_{l}}=y_{j_{l}} \in R_{j_{l}}$ stays bounded. In this case we replace the point $\left(d, z_{1}, \cdots, z_{r}\right)$ by the point in which the $z_{j_{l}}$ coordinate is replaced by 1 . This results in a simpler pair of sequences and their divergence is equivalent to the divergence of the original pair. We may thus assume that either $y_{j_{l}}$ goes to infinity or that all $y_{j}$ and $y_{j}^{\prime}$-coordinates are 1 .

First consider the case when $y_{j_{l}} \rightarrow \infty$. We will argue that the two sequences diverge. It will not be important that the $x$-coordinates on the two sides belong to disjoint simplices, so we will collect all $x$ 's and $x^{\prime \prime}$ s into an element denoted $u$, and we will let $s$ be the difference between the two elements, so we write:

$$
u d y_{j_{1}} \cdots y_{j_{l}}=d^{\prime} y_{k_{1}}^{\prime} \cdots y_{k_{q}}^{\prime} s
$$

where we assume, by way of contradiction, that the sequence $s$ is bounded.

Consider the adjoint representation of $G$ on $A u t(\mathfrak{g})$. We will obtain a contradiction by comparing the automorphisms of $\mathfrak{g}$ the two sides induce. 
We label the roots $\hat{\alpha}_{j_{1}}, \hat{\alpha}_{j_{2}}, \cdots, \hat{\alpha}_{j_{l}}$ by "D" and $\hat{\alpha}_{k_{1}}, \hat{\alpha}_{k_{2}}, \cdots, \hat{\alpha}_{k_{q}}$ by "U". Lemma 10.3 (with $\hat{\alpha}=\hat{\alpha}_{j_{l}}$ ) provides us with $\sigma, \mu \in \Phi \cup\{0\}$. We will focus on the $\mathfrak{g}_{\mu} \rightarrow \mathfrak{g}_{\sigma}$ component of the transformation (2). This component will play the role of a "matrix position" in our examples.

First, we look at $u d y_{j_{1}} \cdots y_{j_{l}}$. Lemma 10.4(1) and Lemma 10.5 imply that $A d\left(y_{j_{l}}\right)$ maps $\mathfrak{g}_{\mu}$ into

$$
\mathfrak{g}_{\mu} \oplus \mathfrak{g}_{\mu-\hat{\alpha}_{j_{l}}} \oplus \mathfrak{g}_{\mu-2 \hat{\alpha}_{j_{l}}} \oplus \cdots
$$

which, by Lemma 10.3(1) and (2) is just $\mathfrak{g}_{\mu} \oplus \mathfrak{g}_{\sigma}$, and further, $\operatorname{Ad}\left(y_{j_{l}}\right)=I \oplus$ $\left[t \zeta_{\hat{\alpha}_{j_{l}}}, \cdot\right]$ on $\mathfrak{g}_{\mu}$. By Lemma 10.3 $(2)$ we have

$$
A d\left(y_{j_{1}} y_{j_{2}} \cdots y_{j_{l-1}}\right) \mid \mathfrak{g}_{\sigma}=I
$$

and

$$
A d\left(y_{j_{1}} y_{j_{2}} \cdots y_{j_{l-1}}\right) \mid \mathfrak{g}_{\mu}
$$

has its image contained in

$$
\oplus\left\{\mathfrak{g}_{\phi} \mid \mu-\phi \text { is a nonnegative linear combination of } \hat{\alpha}_{j_{1}}, \cdots, \hat{\alpha}_{j_{l-1}}\right\} \text {. }
$$

Next, $A d(d)$ preserves each $\mathfrak{g}_{\phi}$ and $A d(u)$ maps $\mathfrak{g}_{\phi}$ into

$$
\bigoplus_{\nu-\phi \in \Phi+\cup\{0\}} \mathfrak{g}_{\nu}
$$

We now claim that $A d(u)$ has $\mathfrak{g}_{\sigma}$-component equal to 0 when restricted to (3). Otherwise, we get equations:

$$
\begin{aligned}
\mu-\phi & =a_{1} \hat{\alpha}_{j_{1}}+\cdots+a_{l-1} \hat{\alpha}_{j_{l-1}} \\
\sigma-\phi & =b_{1} \hat{\alpha}_{1}+\cdots+b_{r} \hat{\alpha}_{r}
\end{aligned}
$$

with all coefficients $\geq 0$ which, together with $\mu-\sigma=\hat{\alpha}_{j_{l}}$, imply (by subtracting the second and third from the first) that

$$
b_{1} \hat{\alpha}_{1}+\cdots+b_{r} \hat{\alpha}_{r}=a_{1} \hat{\alpha}_{j_{1}}+\cdots+a_{l-1} \hat{\alpha}_{j_{l-1}}-\hat{\alpha}_{j_{l}}
$$

thus violating the linear independence of the simple roots.

Summarizing, we have

Lemma 11.1. The $\mathfrak{g}_{\mu} \rightarrow \mathfrak{g}_{\sigma}$-component of $A d\left(u d y_{j_{1}} \cdots y_{j_{l}}\right)$ is

$$
D_{\sigma} \circ\left[t \zeta_{\hat{\alpha}_{j_{l}}}, \cdot\right]
$$

where $D_{\sigma}$ is the restriction of $A d(d)$ to $\mathfrak{g}_{\sigma}$. 
We now perform a similar analysis for the right hand side of (2).

We have that $A d\left(d^{\prime}\right)$ preserves each root space. By Lemma 10.3 (3), the only root space $\mathfrak{g}_{\phi}$ such that $A d\left(y_{k_{1}}^{\prime} \cdots y_{k_{q}}^{\prime}\right)$ has a nontrivial $\mathfrak{g}_{\sigma}$-component when restricted to it is $\mathfrak{g}_{\sigma}$ and the component is $I$. We therefore have

Lemma 11.2. The $\mathfrak{g}_{\mu} \rightarrow \mathfrak{g}_{\sigma}$-component of $A d\left(d^{\prime} y_{k_{1}}^{\prime} \cdots y_{k_{q}}^{\prime} s\right)$ is

$$
D_{\sigma}^{\prime} S_{\mu \sigma}
$$

where $D_{\sigma}^{\prime}$ is the restriction of $A d\left(d^{\prime}\right)$ to $\mathfrak{g}_{\sigma}$ and $S_{\mu \sigma}$ is the $\mathfrak{g}_{\mu} \rightarrow \mathfrak{g}_{\sigma}$-component of $\mathrm{Ad}(s)$.

We now conclude that

$$
D_{\sigma} \circ\left[t \zeta_{\hat{\alpha}_{j_{l}}}, \cdot\right]=D_{\sigma}^{\prime} S_{\mu \sigma}
$$

i.e.

$$
D \circ\left[t \zeta_{\hat{\alpha}_{j_{l}}}, \cdot\right]
$$

is a bounded sequence of maps as $t \rightarrow \infty$ and $D$ represents a sequence in $M_{\mathbb{R}}$. Let $L$ be a (rational) lattice in $\mathfrak{g}_{\sigma}$ that contains a nonzero vector $h$ in the image of the map $\left[\zeta_{\hat{\alpha}_{j_{l}}}, \cdot\right]$. Lemma 10.6 yields a subgroup $\Gamma$ of finite index in $M_{\mathbb{Z}}$ such that $\operatorname{Ad}(\Gamma)$ preserves $L$. Since $\Gamma$ is cocompact in $M$, it follows that $A d(M)$ nearly preserves $L$ in the sense that there is a neighborhood $\Omega$ of $0 \in \mathfrak{g}_{\sigma}$ such that $\operatorname{Ad}(M)$ sends no nonzero element of $L$ (in particular, $h$ ) into $\Omega$ (see Mahler's criterion, Rag72, Corollary 10.9]). It follows that $A d(M)$ sends th outside $t \Omega$, so the composition $D \circ\left[t \zeta_{\hat{\alpha}_{j_{l}}}, \cdot\right]$ is not a uniformly bounded map as $t \rightarrow \infty$. This contradiction proves Lemma $\mathrm{B}$ under our original assumption that $y_{j_{l}} \rightarrow \infty$.

Now suppose that all $y_{j}$ and $y_{j}^{\prime}$ coordinates are 1. Equation (2) becomes

$$
u d=d^{\prime} s
$$

This is an equation in the minimal parabolic subgroup $P=Z(S)^{0} \cdot U$. After passing to a finite cover, we may assume that $P=Z(S)^{0} \rtimes U$. Consider the canonical homomorphism $\tau: P \rightarrow Z(S)^{0}$. It maps $u d$ to $d$ and $d^{\prime} s$ to $d^{\prime} s^{\prime}$ where $s^{\prime}$ is a bounded sequence in $M_{\mathbb{R}}$. Thus $d$ and $d^{\prime}$ stay a bounded distance apart. Since by construction they belong to divergent cones in $M_{\mathbb{R}}$, they both have to stay bounded. It follows that $u$ must stay bounded, i.e. that the sequence $x$ and the sequence $x^{\prime}$ stay within bounded distance. But by Corollary 2.2 this means that both $x$ and $x^{\prime}$ stay bounded, contradicting the assumption that the original sequences were chosen to be unbounded. This contradiction proves Lemma B. 
Proof of Lemma 10.3. Every root can be written as a linear combination of simple roots. The key to this proof is the fact that the coefficients in such linear combinations are explicitly known (and can be found e.g. in Kna96, Appendix C]).

We first note that if $\Phi$ is not irreducible, then the statement follows immediately from the corresponding statements for the irreducible components. The reason for this is that $\Phi$ is then the disjoint union of its irreducible components, and any ordering of $\hat{\Delta}$ that restricts correctly to each component will work. Moreover, if there are elements of $\hat{\Delta}$ that are not labeled at all (which is the case when $\hat{\alpha}$ is not the highest node) then we may restrict our consideration to the root system $\Phi^{\prime}$ generated by the labeled nodes (and in fact to the irreducible component of $\Phi^{\prime}$ that contains $\hat{\alpha}$ ).

Here is one situation when we can take $\sigma$ to be the negative of the largest positive root: $\sigma+\hat{\alpha} \in \Phi \cup\{0\}$ and if we write $\sigma=-m \hat{\alpha}-\cdots$ as a linear combination of simple roots, then all other roots in $\Phi$ have their $\hat{\alpha}$-coefficient $>-m$. These situations, together with the coefficients, are pictured below, with the node $\hat{\alpha}$ circled.

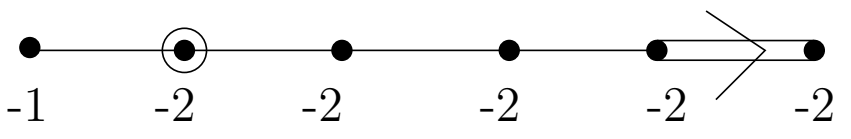

Figure $3: B_{n}, n \geq 3$

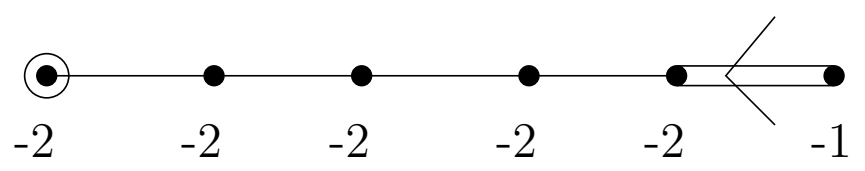

Figure 4: $C_{n}, n \geq 2$

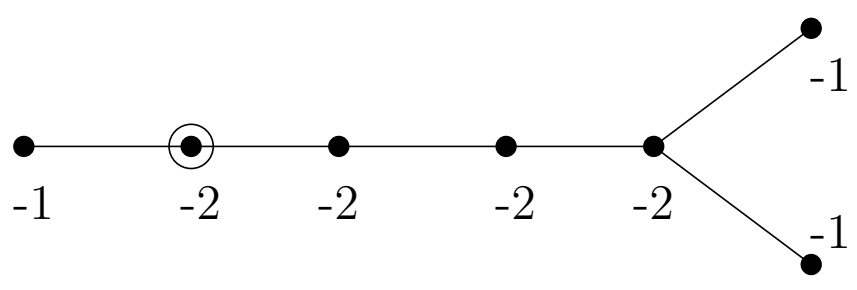

Figure 5: $D_{n}, n \geq 4$ 

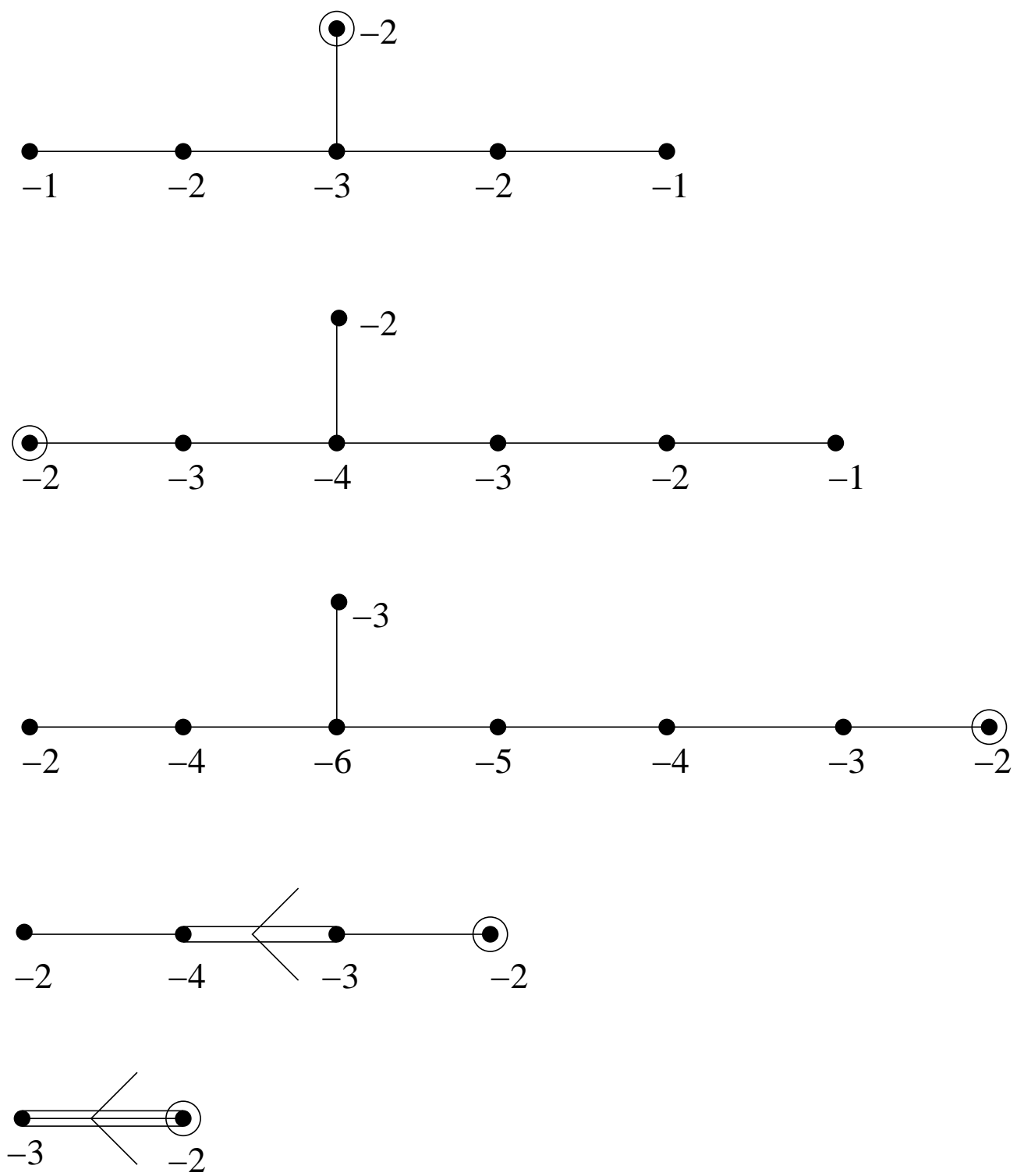

Figure 6: Exceptional reduced root systems $E_{6}, E_{7}, E_{8}, F_{4}, G_{2}$ 


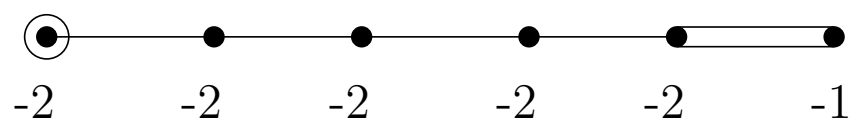

Figure 7: Unreduced root system $B C_{n}, n \geq 2$. The positive roots are $e_{i} \pm e_{j}$ for $i<j, e_{i}, 2 e_{i}, i=1,2, \cdots, n$. The nodes correspond to the roots (from left to right) $e_{1}-e_{2}, e_{2}-e_{3}, \cdots, e_{n-1}-e_{n}, e_{n}, 2 e_{n}$ with the last two corresponding to the same (rightmost) node in the diagram. The largest root is $2 e_{1}$ and its coefficients with respect to the nodes are $2,2, \cdots, 2,1$ if we take $\hat{\alpha}_{n}=2 e_{n}$ as the representative of the last node.

In the case of $B C_{1}$ (i.e. $\Phi=\{-2 \alpha,-\alpha, \alpha, 2 \alpha\}$ ) we can take $\sigma=-2 \alpha=-\hat{\alpha}$ and $\mu=0$. This leaves us with type $A_{n}$. The ordering is the usual linear ordering of the nodes. Say $\alpha$ is node $k$ in this ordering and let a labeling by "U"'s and "D"'s of nodes $\leq k$ be given as in the lemma. Let $1 \leq l \leq k$ be such that the label of node $l$ is " $\mathrm{D}$ " but the label of node $l-1$ is not " $\mathrm{D}$ " (or $l=1$ ). Define $\sigma$ as the negative of the sum of the nodes $l, l+1, \cdots, k$.

In general, to define the ordering on $\hat{\Delta}$, we follow this procedure: Work separately on components. If a component is of type $A_{n}$ order the nodes linearly. Otherwise, define the highest root in the ordering to be the circled node in the corresponding figure above. Then pass to the subdiagram consisting of unlabeled nodes and repeat the procedure.

We finish the paper by looking at few more examples.

Remark 5. The ordering of $\Delta$ was used in two places: to define $\Phi_{i}^{+}$(and the associated nilpotent groups $N_{i}$ ) and in determining the order in which the $y_{i}$ 's come in the definition of the map $\Psi$. The reader will observe that we can use two different orders on $\Delta$ : an arbitrary order to define $\Phi_{i}^{+}$and the one from Lemma 10.3 to order the $y_{i}$ 's. It is convenient to use the standard order for the first purpose since then the $N_{i}$ 's are standard nilpotent matrix groups. This is the practice we follow in the examples.

\section{$12 \Gamma=S p_{2 n}(\mathbb{Z})$}

Consider $V=\mathbb{C}^{2 n}$ with the standard $\mathbb{C}$-basis $e_{1}, e_{2}, \cdots, e_{n}, e_{n+1}, \cdots, e_{2 n}$. Let $J$ be the anti-symmetric bilinear pairing defined by $J\left(e_{i}, e_{n+i}\right)=1$ and $J\left(e_{i}, e_{j}\right)=$ 0 if $|j-i| \neq n$. The group $S p_{2 n}(\mathbb{C})$ is the subgroup of $G L_{2 n}(\mathbb{C})$ consisting of matrices that preserve $J$. It is convenient to represent the matrices in $S p_{2 n}(\mathbb{C})$ 
and in its Lie algebra in $2 \times 2$ block form corresponding to the partition of the basis for $V$ into the first $n$ and last $n$ vectors. Thus $J$ is represented by $J=\left(\begin{array}{cc}0 & -I \\ I & 0\end{array}\right)$. The Lie algebra consists of block matrices $X=\left(\begin{array}{cc}A & B \\ C & D\end{array}\right)$ such that $X J+J X^{t}=0$, i.e. $B$ and $C$ are symmetric matrices, and $D$ is the negative transpose of $A$. A maximal split torus $S$ can be taken to consist of diagonal matrices

$$
\operatorname{diag}\left[s_{1}, s_{2}, \cdots, s_{n}, s_{1}^{-1}, s_{2}^{-1}, \cdots, s_{n}^{-1}\right]
$$

Denote by $y_{i}: S \rightarrow \mathbb{C}$ the character that takes the above matrix to $s_{i}$. The positive roots are

$$
y_{i}-y_{j},(i<j) ; y_{i}+y_{j},(i \neq j) ; 2 y_{i}
$$

and the simple roots are $y_{1}-y_{2}, y_{2}-y_{3}, \cdots, y_{n-1}-y_{n}, 2 y_{n}$, so the root system is of type $C_{n}$. The Lie algebra $\mathfrak{n}_{i}$ for $i<n$ consists of matrices that in the column $i+1$ of the (1,1)-block have arbitrary entries above the diagonal and 0 in all other positions, the $(2,2)$-block is the negative transpose of the $(1,1)$-block, and the $(1,2)$ and $(2,1)$-blocks are 0 . For $i=n$ the Lie algebra $\mathfrak{n}_{n}$ consists of matrices $\left(\begin{array}{ll}0 & B \\ 0 & 0\end{array}\right)$ with $B$ symmetric. In this case $\Phi_{n}^{+}$consists of the roots in classes 2 and 3 above. The obstructor complex in this case is

$$
L=S_{+}^{0} * S_{+}^{1} * \cdots * S_{+}^{n-2} * S_{+}^{\frac{(n+2)(n-1)}{2}}
$$

The Lie algebras $\mathfrak{n}_{i}$ are abelian and exponentiation (to obtain $N_{i}$ ) amounts to adding the identity matrix.

\section{$13 \Gamma=S p_{2 n}(\mathcal{O})$}

If $r$ and $s$ denote the numbers of real and complex places, then $\mathcal{O} \cong \mathbb{Z}^{r+2 s}$ as an abelian group so the dimensions of $N_{i}$ 's above should be multiplied by $r+2 s$. The centralizer $Z(S)$ consists of the diagonal matrices (5) and $M_{\mathbb{Z}}$ is commensurable with the group of such matrices with entries in $\mathcal{O}^{*}$ : it is an abelian group of rank $(n-1)(r+2 s)$. The obstructor complex is thus

$$
S^{(n-1)(r+2 s)-1} * S_{+}^{r+2 s-1} * S_{+}^{2(r+2 s)-1} * \cdots * S_{+}^{(n-1)(r+2 s)-1} * S_{+}^{\frac{n(n+1)(r+2 s)}{2}-1}
$$

\section{$14 \Gamma=S O(Q)$ for a nondegenerate form $Q$}

Any nondegenerate quadratic form $Q$ defined over $\mathbb{Q}$ on a vector space $V$ can be represented as a direct sum of a certain number, say $q$, of hyperbolic planes 
and of an anisotropic quadratic form $Q_{0}$ (i.e. $Q_{0}$ does not take value 0 on nonzero rational vectors). We follow notation from [Bor91, V.23.4], where the reader can find more details about $S O(Q)$. Choose a rational basis $e_{1}, e_{2}, \cdots, e_{n}$ of $V$ so that $e_{i}, e_{n-q+i}$ span a hyperbolic direct summand for $i=1,2, \cdots, q$ (with $<e_{i}, e_{i}>=<e_{n-q+i}, e_{n-q+i}>=0,<e_{i}, e_{n-q+i}>=1$ in the associated symmetric pairing) and $e_{q+1}, \cdots, e_{n-q}$ span the $Q_{0}$-summand. The maximal split $\mathbb{Q}$-torus $S$ in $S O(Q)$ is the group of diagonal matrices

$$
\operatorname{diag}\left[s_{1}, s_{2}, \cdots, s_{q}, 1,1, \cdots, 1, s_{1}^{-1}, s_{2}^{-1}, \cdots, s_{q}^{-1}\right]
$$

Denote by $y_{i}: S \rightarrow \mathbb{C}$ the character that sends the above matrix to $s_{i}$. The centralizer $Z(S)$ is $S \times S O\left(Q_{0}\right)$ and the positive roots are

$$
y_{i}-y_{j},(i<j) ; y_{i}+y_{j},(i \neq j) ; y_{i}
$$

with the first two kinds having multiplicity 1 and the third kind of multiplicity $n-2 q$ (so the third kind is not present when $n=2 q$ ). The simple roots are $y_{1}-y_{2}, y_{2}-y_{3}, \cdots, y_{q-1}-y_{q}, y_{q}$ which is type $B_{q}$ (if $n=2 q$ the type is $D_{q}$ ). Every element of $S O(Q)$ and of its Lie algebra is conveniently represented in a $(3,3)$-block form corresponding to first $q$, middle $n-2 q$, and last $q$ basis vectors. A block matrix $\left(A_{i j}\right)$ is in the Lie algebra of $S O(Q)$ if and only if the following 6 conditions are satisfied:

$$
\begin{aligned}
A_{13}+A_{13}^{t} & =A_{12} F_{0}+A_{23}^{t}=A_{11}+A_{33}^{t}=0 \\
A_{22} F_{0}+F_{0} A_{22}^{t} & =A_{32} F_{0}+A_{21}^{t}=A_{31}+A_{31}^{t}=0
\end{aligned}
$$

where $F_{0}$ is the matrix of $Q_{0}$.

We now describe the Lie algebras $\mathfrak{n}_{i}$ of the groups $N_{i}, i=1,2, \cdots, q-1$ (we are using the standard ordering of the simple roots): In the (1,1)-block consider the $i$ positions above the diagonal in column $i+1$. Any (complex) entries are allowed. Then change the sign of these entries and transpose, and write this row vector in the $3 \times 3$ block in row $i+1$ to the left of the diagonal. To get $N_{i}$ exponentiate - in this case this amounts to adding the identity matrix. Intersecting with real points, this defines the space $C_{i}$ from the proof of Lemma $\mathrm{B}-$ it is the cone on the sphere of dimension $i-1$.

The group $N_{q}$ is not abelian - it is 2-step nilpotent. Its Lie algebra is spanned by the positive roots in classes 2 and 3 above and it consists of block matrices that have vanishing all 3 diagonal blocks and all 3 blocks below the diagonal. The group of real points of $N_{q}$ has dimension $q(n-2 q)+(1+2+$ $\cdots+(q-1))$.

The ray corresponding to the root $y_{i}-y_{i+1}$ is obtained by exponentiating matrices with entry $t \geq 0$ in position $(i+1, i)$ of $A_{11}$ and entry $-t$ in position 
$(i, i+1)$ of $A_{33}$ - in this case exponentiation amount to adding the identity matrix. To define a ray corresponding to the root $y_{q}$ choose a nonzero rational row vector $v$ of length $n-2 q$ and place it in the last row of $A_{12}$ and then place the column vector $-F_{0} v^{t}$ in the last column of $A_{23}$. The ray is obtained by exponentiating positive multiples of this matrix - this amounts to adding the identity matrix and the entry $-\frac{1}{2} v F_{0} v^{t}$ in position $(q, q)$ of $A_{13}$.

If the form $Q_{0}$ is definite, then $M_{\mathbb{R}}=S O\left(Q_{0}\right)_{\mathbb{R}}$ is a compact group and $C_{M}$ will be a point. If $Q_{0}$ is not definite, then $C_{M}$ can be identified with the symmetric space of $S O\left(Q_{0}\right)_{\mathbb{R}}$.

\section{References}

[Ash77] Avner Ash, Deformation retracts with lowest possible dimension of arithmetic quotients of self-adjoint homogeneous cones, Math. Ann. 225 (1977), no. 1, 69-76.

[BH99] Martin R. Bridson and André Haefliger, Metric spaces of non-positive curvature, Springer-Verlag, Berlin, 1999.

[BHC62] Armand Borel and Harish-Chandra, Arithmetic subgroups of algebraic groups, Ann. of Math. (2) 75 (1962), 485-535.

[BKK] Mladen Bestvina, Michael Kapovich, and Bruce Kleiner, Van Kampen's embedding obstruction for discrete groups, preprint, 2000.

[Bor69] Armand Borel, Introduction aux groupes arithmétiques, Hermann, Paris, 1969, Publications de l'Institut de Mathématique de l'Université de Strasbourg, XV. Actualités Scientifiques et Industrielles, No. 1341.

[Bor91] Armand Borel, Linear algebraic groups, second ed., Springer-Verlag, New York, 1991.

[BS73] A. Borel and J.-P. Serre, Corners and arithmetic groups, Comment. Math. Helv. 48 (1973), 436-491, Avec un appendice: Arrondissement des variétés à coins, par A. Douady et L. Hérault.

[BW80] Armand Borel and Nolan R. Wallach, Continuous cohomology, discrete subgroups, and representations of reductive groups, Princeton University Press, Princeton, N.J., 1980.

[FH91] William Fulton and Joe Harris, Representation theory, SpringerVerlag, New York, 1991, A first course, Readings in Mathematics. 
[Got48] Morikuni Gotô, Faithful representations of Lie groups. I, Math. Japonicae 1 (1948), 107-119.

[GS92] Mikhail Gromov and Richard Schoen, Harmonic maps into singular spaces and p-adic superrigidity for lattices in groups of rank one, Inst. Hautes Études Sci. Publ. Math. (1992), no. 76, 165-246.

[Hel78] Sigurdur Helgason, Differential geometry, Lie groups, and symmetric spaces, Academic Press Inc. [Harcourt Brace Jovanovich Publishers], New York, 1978.

[Hoc65] G. Hochschild, The structure of Lie groups, Holden-Day Inc., San Francisco, 1965.

[Iwa49] Kenkichi Iwasawa, On some types of topological groups, Ann. of Math. (2) 50 (1949), 507-558.

[Kna96] Anthony W. Knapp, Lie groups beyond an introduction, Birkhäuser Boston Inc., Boston, MA, 1996.

[Mal45] A. Malcev, On the theory of the Lie groups in the large, Rec. Math. [Mat. Sbornik] N. S. 16(58) (1945), 163-190.

[Mil76] John J. Millson, On the first Betti number of a constant negatively curved manifold, Ann. of Math. (2) 104 (1976), no. 2, 235-247.

[Mos55] G. D. Mostow, Self-adjoint groups, Ann. of Math. (2) 62 (1955), 4455 .

[Rag72] M. S. Raghunathan, Discrete subgroups of Lie groups, SpringerVerlag, New York, 1972, Ergebnisse der Mathematik und ihrer Grenzgebiete, Band 68.


jecture for actions by Lipschitz maps, Math. Ann. 308 (1997), no. 2, 361-364.

[Zim84] Robert J. Zimmer, Ergodic theory and semisimple groups, Birkhäuser Verlag, Basel, 1984. 